\title{
Large eddy simulation of flame extinction in a turbulent line fire exposed to air-nitrogen co-flow
}

\author{
S. Vilfayeau, J. White, P. Sunderland, A. Marshall, A. Trouvé* \\ Department of Fire Protection Engineering, University of Maryland, College Park, MD \\ 20742, USA
}

\begin{abstract}
The general objective of this project is to support the development and validation of large eddy simulation (LES) models used to simulate the response of fires to the activation of suppression systems. The focus here is on suppression by gaseous agents. The present experimental configuration is a two-dimensional, plane, buoyancy-driven, methane-fueled, turbulent diffusion flame with a controlled co-flow. The co-flow is an air-nitrogen mixture with variable oxygen dilution conditions, including conditions that lead to full flame extinction. Experimental measurements include the global combustion efficiency and global radiative loss fraction. The numerical simulations are performed with a LESbased fire model developed by FM Global and called FireFOAM. In this study, FireFOAM is modified to include a flame extinction model based on the concept of a critical flame Damköhler number and a flame reignition model based on the concept of a critical gas temperature. The numerical simulations are found to successfully reproduce the rapid change that is observed experimentally when exposing the flame to a co-flow with decreasing oxygen strength: the change corresponds to an abrupt transition from a strong flame with a global combustion efficiency close to one to a residual flame with a global combustion efficiency close to zero.
\end{abstract}

Keywords: Buoyant turbulent diffusion flame, Flame extinction, Fire

\footnotetext{
* Corresponding author

Email address: atrouve@umd.edu (A. Trouvé)
}

Preprint submitted to Fire Safety Journal

September 28, 2016

(C) 2016. This manuscript version is made available under the Elsevier user license http://www.elsevier.com/open-access/userlicense/1.0/ 
suppression, Large Eddy Simulation, FireFOAM

\section{Introduction}

Computational fluid dynamics (CFD) modeling has emerged over the past two decades as a powerful tool for both research-level and engineering-level projects in the area of fire safety. One of the main technical challenges found

5 in a CFD treatment of compartment fires is the description of partial or total flame extinction, as may occur in under-ventilated fire configurations or in configurations in which a fire suppression system is activated (e.g., systems based on the injection of inert gas or injection of a water spray). This challenge is particularly difficult because flame extinction corresponds to phenomena in which the effects of finite-rate combustion chemistry become a dominant factor: turbulent combustion models used in fire applications typically ignore combustion chemistry (they assume that the rate of combustion is determined by the turbulent rate of fuel-air mixing) and are therefore ill-prepared to simulate flame extinction phenomena.

Available models used to describe flame extinction in fire problems are based on the concepts of a critical flame temperature $[1,2]$ or a critical flame Damköhler number [3-7]. Models based on the concept of a critical flame temperature choose to ignore the importance of chemical time scales and are not consistent with known laminar flame phenomenology [8]. Models based on the concept of a critical flame Damköhler number explicitly or implicitly account for at least one chemical time scale, are consistent with known laminar flame phenomenology, and therefore may be expected to be more accurate [8]. The occurrence of flame extinction is also followed by that of reignition and the modeling of under-ventilated fires or fire suppression requires both an extinc-

25 tion model and a reignition model [4], a difficulty that is generally overlooked in the fire modeling literature.

The objective of the present study is to evaluate the performance of current CFD-based fire models in their treatment of flame extinction and reignition. 
The study considers an experimental configuration developed at the University of Maryland (UMD) $[9,10]$ and corresponding to a buoyant, turbulent, methanefueled line fire exposed to air-nitrogen mixtures of variable oxygen strength. The numerical solver is a large eddy simulation (LES) solver developed by FM Global and called FireFOAM [11]. The present study is a continuation of previous work [4]: the extinction/reignition models in Ref. [4] were first formulated using the concept of mixture fraction; these models were then applied to the case of reduced-scale compartment fires with extinction resulting from underventilation. The extinction/reignition models in the present study have been re-formulated to a more general framework that does not use mixture fraction; these models are now applied to the UMD line fire experiment characterized by 40 well-controlled experimental conditions and a more elaborate instrumentation.

In the following, the experimental configuration is described in section 2.1; the numerical solver, including a description of the new flame extinction and reignition models, in section 2.2; and the numerical configuration in section 2.4. Results on the simulated flame structure are presented in section 3.1; on grid convergence in section 3.2; on angular space discretization in section 3.3; and on flame radiative emissions in section 3.4. Numerical results are compared to experimental data in sections 3.5 and 3.6. Section 3.5 uses preliminary experimental data on local variations of temperature and oxygen mole-fraction. Section 3.6 uses recently obtained experimental data on global combustion effi50 ciency.

\section{Experimental configuration, numerical solver and numerical con- figuration}

\subsection{Experimental configuration}

The configuration adopted in the present study corresponds to a buoyant, turbulent, methane-fueled diffusion flame with a controlled co-flowing oxidizer $[9,10]$. Flames are stabilized above a 5 -cm-wide by 50-cm-long slot burner surrounded by a $15-\mathrm{cm}$-wide by $60-\mathrm{cm}$-long plate of ceramic fiberboard and further 


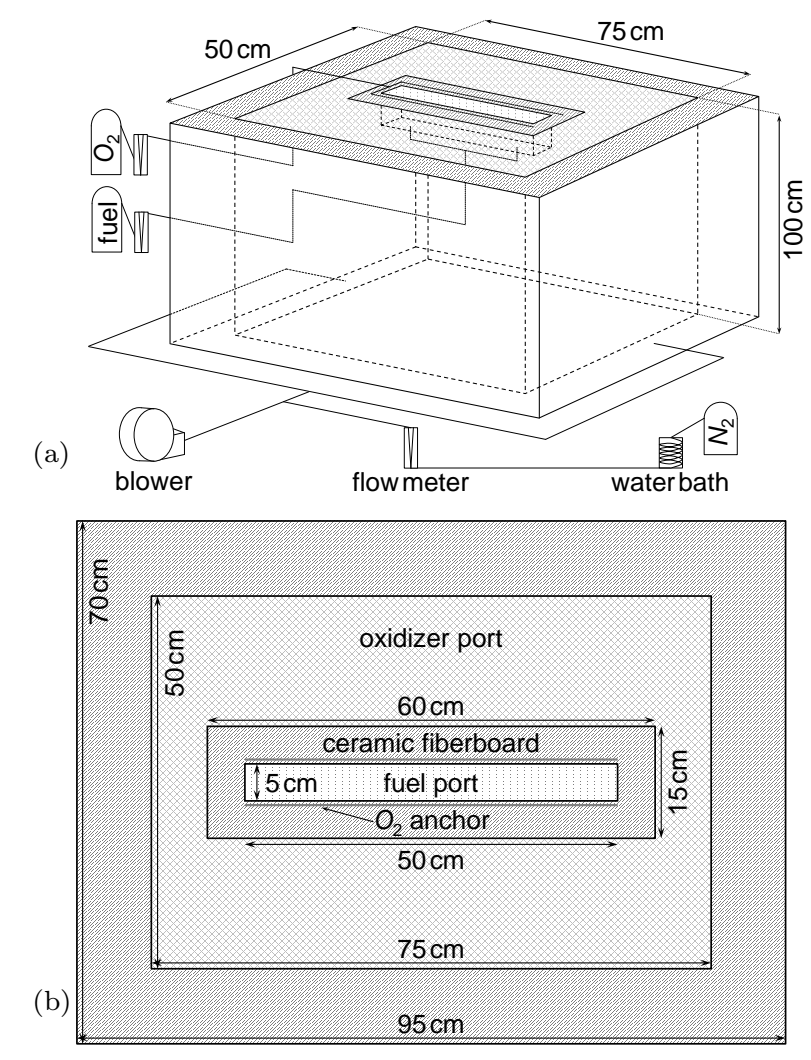

Figure 1: (a) Diagram of experimental facility. (b) Top-view of burner/co-flow outlet. Reproduced from [9].

surrounded by a 50 -cm-wide by 75 -cm-long oxidizer co-flow (see Fig. 1). The plate adds a horizontal component to the flow motion near the flame base and 60 was introduced in previous work with the intent to promote faster transition to fully-turbulent flow conditions [9]. The methane velocity is $6.0 \mathrm{~cm} / \mathrm{s}$, corresponding to a mass flow rate of $1.0 \mathrm{~g} / \mathrm{s}$, and a nominal total heat release rate of $50 \mathrm{~kW}$ for the unsuppressed flame.

In the baseline unsuppressed flame configuration, the oxidizer co-flow is made 65 of pure air; the air velocity is $20.0 \mathrm{~cm} / \mathrm{s}$, corresponding to a mass flow rate of $68.5 \mathrm{~g} / \mathrm{s}$ or approximately four times the stoichiometric requirement of the 
methane flow. Note that this flow rate is too low to supply all of the gas entrained by the flame and that while the base of the flame is exclusively exposed to the co-flow, the tip region of the flame is exposed to entrained air from the open ambient. While an undesirable feature, numerical tests suggest that this effect remains limited and that the heat release rate associated with the uncontrolled entrained ambient air corresponds to less than $5 \%$ of the total heat release rate of the flame [12].

In suppressed flame configurations, the air flow rate remains fixed and nitrogen is added to the co-flow with a mass flow rate between $0-60 \mathrm{~g} / \mathrm{s}$; the oxygen mole-fraction in the co-flow, noted $X_{O_{2}}$, varies between $21 \%$ and $11 \%$. Note that a discrepancy between the numerical and experimental configurations was recently discovered: the numerical configuration follows the protocol described above in which the air mass flow rate is fixed while the nitrogen and total (air plus nitrogen) mass flow rates are variable; in contrast, the experimental configuration follows a slightly different protocol in which both the air and nitrogen mass flow rates are variable while the total mass flow rate is fixed (and equal to $85 \mathrm{~g} / \mathrm{s})$. Recent numerical tests suggest that this discrepancy negligibly affects the presently reported results.

An additional small co-flow stream of pure oxygen, called the oxygen anchor, is introduced along the length of the burner to strengthen the base of the flame and oppose liftoff extinction [9]. The velocity of the oxygen anchor is $1.2 \mathrm{~cm} / \mathrm{s}$, corresponding to a mass flow rate of $0.08 \mathrm{~g} / \mathrm{s}$, and a nominal anchor-limited total heat release rate of $1 \mathrm{~kW}$ or $2 \%$ of the size of the unsuppressed flame.

Flame suppression is characterized through a variety of diagnostics including measurements of the global combustion efficiency using $\mathrm{CO}_{2}$ generation and $\mathrm{O}_{2}$ consumption calorimetry [10], measurements of the global radiative loss fraction using a heat flux transducer combined with time-resolved infrared camera imaging and a multipoint radiation source model [9], measurements of local gas

95 temperature using exposed-junction, $1.0 \mathrm{~mm}$ bead-diameter K-type thermocouple probes (uncertainty $\pm 2 \mathrm{~K}$; response time $\sim 3 \mathrm{~s}$ ) and measurements of local oxygen mole-fraction using a sampling probe connected to a Servomex 540E 
paramagnetic oxygen-analyzer (uncertainty $\pm 1250 \mathrm{ppm}$; response time $\sim 5 \mathrm{~s}$ )

[9]. For calorimetry measurements, combustion products are collected in an

exhaust duct, where a gas sampling system provides measurement of the molefractions of $\mathrm{O}_{2}, \mathrm{CO}_{2}, \mathrm{CO}$ and $\mathrm{H}_{2} \mathrm{O}$. From these measurements, the global heat release rate $( \pm 1.5 \mathrm{~kW})$ is derived via mass conservation analysis (for a detailed description of this measurement, the reader is referred to Ref. [10]). Combustion efficiency $( \pm 3 \%)$ is determined by dividing the calorimetry-derived heat release rate by the nominal total heat release rate of the flame $(50 \mathrm{~kW})$, defined as the product of the measured fuel mass flow rate times the theoretical heat of combustion. The combustion efficiency is a function of the oxygen mole-fraction in the co-flow. Global flame extinction is observed at $X_{O_{2}, \text { ext }}=12.2 \% \pm 0.2 \%$.

\subsection{Numerical solver}

FireFOAM $[11,13,14]$ is based on OpenFOAM [15], an open-source generalpurpose CFD software package. FireFOAM is an object-oriented, $\mathrm{C}++$ based, second-order accurate, finite volume solver with implicit time integration; PISO and SIMPLE types of solution procedures are used to couple sequentially-solved equations; the solver features advanced meshing capabilities (the mesh geometry

is a structured or unstructured polyhedral mesh); it also features a massively parallel computing capability using Message Passing Interface (MPI) protocols.

FireFOAM uses a Favre-filtered compressible-flow LES formulation and provides a choice between several modeling options for the treatment of turbulence, combustion, and thermal radiation. In the present study, subgrid-scale (SGS) turbulence is described using the one-equation eddy viscosity model (a model based on solving a transport equation for SGS turbulent kinetic energy or TKE) [16]. Combustion is described using the classical concept of a global combustion equation combined with the eddy dissipation concept (EDC) model [17]. Thermal radiation is described by solving the radiative transfer equation (RTE). Radiative properties are treated as spectrally-averaged quantities and a gray medium is assumed. In FireFOAM, the RTE is solved using a finite volume implementation of the discrete ordinate method (DOM) [18]. 
In the present study, the description of thermal radiation is further simplified by assuming a non-scattering, non-absorbing, optically-thin medium and using the empirical concept of a user-prescribed global radiative loss fraction, $\chi_{r}$. For each simulation, $\chi_{r}=f\left(X_{O_{2}}\right)$ is prescribed from experimental measurements [9]. The assumption of an emitting but non-absorbing medium as well as that of a prescribed radiative loss fraction should be viewed as an intermediate modeling step. Also note that the methane-fueled flames are only weakly sooting and therefore soot is neglected in the present simulations.

\subsection{Flame extinction and reignition models}

The FireFOAM solver has been recently modified to include a flame extinction model based on the concept of a critical flame Damköhler number and a flame reignition model based on the concept of a critical gas temperature $[4,8]$.

The extinction model was initially formulated using the concept of mixture fraction [4]. However, because mixture fraction becomes complicated in problems with multiple fuels and multiple oxidizer streams (these problems require multiple mixture fraction variables), the model has been re-formulated to a more general framework that does not use mixture fraction.

\subsubsection{Modified global combustion scheme}

The combustion model in FireFOAM uses a global combustion equation,

$$
\mathrm{CH}_{4}+2 \mathrm{O}_{2} \rightarrow \mathrm{CO}_{2}+2 \mathrm{H}_{2} \mathrm{O}
$$

which corresponds to a normal combustion step in which fuel (here methane) is oxidized into carbon dioxide and water vapor. When considering extinction, reaction (GR1) is enhanced by two additional steps,

$$
\begin{aligned}
\mathrm{CH}_{4}+2 \mathrm{O}_{2} & \rightarrow\left(\mathrm{CH}_{4}\right)^{*}+2 \mathrm{O}_{2}, \\
\left(\mathrm{CH}_{4}\right)^{*}+2 \mathrm{O}_{2} & \rightarrow \mathrm{CO}_{2}+2 \mathrm{H}_{2} \mathrm{O},
\end{aligned}
$$

where reaction (GR2) describes flame extinction, treated as a transformation of fresh fuel $\mathrm{CH}_{4}$ into non-burning fuel $\left(\mathrm{CH}_{4}\right)^{*}$, and reaction (GR3) describes reignition, treated as a normal combustion step for $\left(\mathrm{CH}_{4}\right)^{*}$. 
The introduction of two distinct combustion steps (GR1) and (GR3) brings flexibility in the model and allows in particular for a separate treatment of extinction and reignition phenomena (see sections 2.3.2 and 2.3.3 below). This separate treatment was motivated by the modeling objective of avoiding spurious re-ignition of the unburnt fuel downstream of the flame zone (for a detailed discussion of spurious reignition, the reader is referred to Ref. [19]). The list of transported species in the combustion model includes $\mathrm{CH}_{4},\left(\mathrm{CH}_{4}\right)^{*}, \mathrm{O}_{2}, \mathrm{CO}_{2}$ and $\mathrm{H}_{2} \mathrm{O}$.

The closure expressions for the chemical reaction rates are based on the EDC model [17]:

$$
\begin{aligned}
& \overline{\overline{\dot{\omega}}_{G R 1}^{\prime \prime \prime}}=(1-F E F)\left(C_{E D C}\left(\frac{\bar{\rho}}{\tau_{t}}\right) \min \left(\tilde{Y}_{C H_{4}}, \frac{\widetilde{Y}_{O_{2}}}{r_{s}}\right)\right), \\
& \overline{\dot{\omega}_{G R 2}^{\prime \prime \prime}}=\quad \operatorname{FEF}\left(C_{E D C}\left(\frac{\bar{\rho}}{\tau_{t}}\right) \min \left(\tilde{Y}_{C H_{4}}, \frac{\widetilde{Y}_{O_{2}}}{r_{s}}\right)\right), \\
& \overline{\dot{\omega}_{G R 3}^{\prime \prime \prime}}=\quad F I F\left(C_{E D C}\left(\frac{\bar{\rho}}{\tau_{t}}\right) \min \left(\widetilde{Y}_{\left(C H_{4}\right)^{*}}, \frac{\widetilde{Y}_{O_{2}}}{r_{s}}\right)\right),
\end{aligned}
$$

where $F E F$ and FIF designate flame extinction and reignition factors; $C_{E D C}$ is a model coefficient $\left(C_{E D C}=4[17]\right) ; \bar{\rho}$ is the (LES-filtered) mass density (in FireFOAM, $\bar{\rho}$ is calculated from the ideal gas law and an estimate of the filtered gaseous mixture molecular weight in which second-order fluctuations are neglected $) ; \tau_{t}$ is the SGS turbulent mixing time scale $\left(\tau_{t}=\left(1 / C_{e}\right) \times\left(\Delta /\left(k_{S G S}\right)^{1 / 2}\right)\right.$, with $C_{e}$ a dynamic model coefficient, $\Delta$ the local grid cell size, and $k_{S G S}$ the subgrid TKE); $\widetilde{Y}_{\mathrm{CH}_{4}}, \widetilde{Y}_{\left(\mathrm{CH}_{4}\right)^{*}}$, and $\widetilde{Y}_{\mathrm{O}_{2}}$ are the (LES-filtered) mass-fractions of fuel, non-burning fuel, and oxygen; and $r_{s}$ is the stoichiometric oxygen-to-fuel mass ratio.

\subsubsection{Flame extinction factor FEF}

Flame extinction is treated via a critical Damköhler number criterion,

$$
F E F=0.5-0.5 \tanh \left(\frac{D a-D a_{c}}{0.02}\right),
$$

where $D a$ is the flame Damköhler number and $D a_{c}$ its critical value at extinction. The flame extinction factor $F E F$ takes values between 0 and 1 and gives a measure of the local probability of flame extinction: $F E F=0$ if $D a \geqslant D a_{c}$ 
(no extinction) and $F E F=1$ if $D a<D a_{c}$ (full extinction). Following previous

work in Ref. [8], we use $D a_{c}=1$ and write:

$$
D a=C \times \frac{\exp \left(T_{a} / T_{s t}\right)}{\chi_{s t}},
$$

where $C$ and $T_{a}$ are model parameters (to be discussed below), $T_{s t}$ is the flame temperature and $\chi_{s t}$ is the rate of fuel-air mixing. The flame-based quantities $T_{s t}$ and $\chi_{s t}$ are obtained via subgrid-scale models described as follows. (note that the flame temperature $T_{s t}$ should not be confused with the grid-resolved temperature $\widetilde{T}: \widetilde{T}$ is a mean temperature spatially-averaged over the volume of the cell; in contrast, $T_{s t}$ is the temperature of the reactive layers typically occupying a small volume inside the cell.)

The flame temperature $T_{s t}$ is treated by an approximate model as a weighted average between the adiabatic flame temperature $T_{s t}^{a d}$ and the mixing temperature $T_{s t}^{m}$, using the deficit enthalpy variable $H_{s t}$ as a weight coefficient:

$$
T_{s t}=\left(1+H_{s t}\right) T_{s t}^{a d}-H_{s t} T_{s t}^{m}
$$

where $T_{s t}^{m}$ is the temperature that would be obtained with pure fuel-air mixing and without combustion (we use $T_{s t}^{m}=293 \mathrm{~K}$ ) and $T_{s t}^{a d}$ is the temperature that would be obtained with adiabatic combustion (i.e., without radiation losses, convective wall losses, or an evaporating water spray). In the present study, the adiabatic flame temperature $T_{s t}^{a d}$ is prescribed using an expression coming from the classical Burke-Schumann solution, $T_{s t}^{a d}=f\left(Y_{O_{2}, 2}\right)$, where $Y_{O_{2}, 2}$ is the oxygen mass-fraction in the air-nitrogen co-flow [8].

In Eq. 4, the deficit enthalpy variable $H_{s t}$ takes values between $(-1)$ and 0 and gives a measure of the magnitude of non-adiabatic effects at the flame location: $H_{s t}=0$ under adiabatic burning conditions and $H_{s t}=-1$ under suppressed conditions. $H_{s t}$ is calculated as

$$
H_{s t}=-\frac{\left(\widetilde{h}_{s}^{a d}-\widetilde{h}_{s}\right)}{\left(\widetilde{h}_{s}^{a d}-\widetilde{h}_{s}^{m}\right)},
$$

where $\widetilde{h}_{s}, \widetilde{h}_{s}^{a d}$, and $\widetilde{h}_{s}^{m}$ are the (LES-filtered) sensible enthalpy, adiabatic sensible enthalpy, and mixing sensible enthalpy, respectively. 
The (LES-filtered) sensible enthalpy $\widetilde{h}_{s}$ is a principal variable in the Firein flame temperature due to the presence of thermal losses. In the present study, thermal losses are only due to emission of radiative energy.

Furthermore, the rate of fuel-air mixing $\chi_{s t}$ in Eq. 3 is described based on a classical closure expression for the LES-filtered scalar dissipation rate, ${ }_{220} \tilde{\chi}=2\left(\alpha+\frac{\nu_{t}}{P r_{t}}\right)|\nabla \widetilde{Z}|^{2}$, where $\widetilde{Z}$ is the mixture fraction. Replacing mixture fraction by its definition in terms of fuel and oxygen mass-fractions gives

$$
\chi_{s t}=2\left(\alpha+\frac{\nu_{t}}{P r_{t}}\right)\left(\frac{r_{s} \nabla \tilde{Y}_{C H_{4}}+r_{s} \nabla \tilde{Y}_{\left(C H_{4}\right)^{*}}-\nabla \widetilde{Y}_{O_{2}}}{r_{s}+Y_{O_{2}, a i r}}\right)^{2},
$$


where $Y_{O_{2}, \text { air }}$ is the oxygen mass-fraction in air, $Y_{O_{2}, \text { air }}=0.233$.

Finally, the Damköhler-number-based flame extinction criterion in Eq. 3 requires two model parameters $C$ and $T_{a}$. These model parameters are fueldependent and are obtained from basic information on the extinction limits of relevant diffusion flames. For instance, for methane fuel, we use the results from a numerical study by Chan et al. [20] where extinction conditions of methane-air laminar counter-flow diffusion flames are calculated using a detailed chemical kinetic mechanism and in the presence of thermal radiation losses. The simulated flames feature two extinction limits: a classical upper limit corresponding to fast fuel-air mixing conditions (i.e., large values of the scalar dissipation rate $\chi_{s t}$ ), where extinction occurs due to insufficient residence time (often called kinetic extinction); and a lower limit corresponding to slow fuel-air mixing conditions (i.e., small values of $\chi_{s t}$ ), where extinction occurs due to thermal radiation losses ${ }_{235}$ (often called radiation extinction). Results from Ref. [20] give the following extinction conditions: $\chi_{s t, e x t}^{U L}=18.4 \mathrm{~s}^{-1}, T_{s t, e x t}^{U L}=1773 \mathrm{~K}$ and $\chi_{s t, e x t}^{L L}=0.029 \mathrm{~s}^{-1}$, $T_{s t, e x t}^{L L}=1353 \mathrm{~K}$, for the upper and lower limits respectively.

Now, following Refs. [21] and [8], we assume that all extinction events correspond to the same crticial value of the Damköhler number, $D a_{c}=1$. This leads to the following system of equations for $C$ and $T_{a}$ :

$$
\begin{aligned}
& 1=C \times \frac{\exp \left(T_{a} / T_{s t, e x t}^{U L}\right)}{\chi_{s t, e x t}^{U L}} \\
& 1=C \times \frac{\exp \left(T_{a} / T_{s t, e x t}^{L L}\right)}{\chi_{s t, e x t}^{L L}}
\end{aligned}
$$

Thus, Eq. 10 provides a system of two equations with two unknowns, which can be readily solved. We get $C=1.9616 \times 10^{10} \mathrm{~s}^{-1}$ and $T_{a}=36856 \mathrm{~K}$. Values of $C$ and $T a$ for other fuels can be found in Ref. [22].

Figure 2 presents the flammability map implied by the Damköhler-numberbased flame extinction model in Eq. 3. As expected, extinction is promoted by large values of flame stretch and low values of flame temperature. Large values of flame stretch are typically associated with high flow turbulence intensities while low values of flame temperature typically result from oxygen dilution effects 


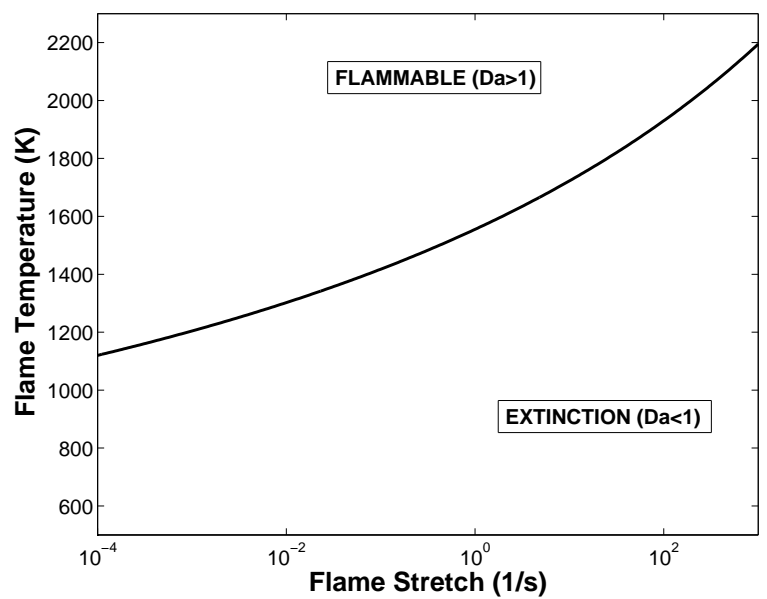

Figure 2: Flammability map for methane-air diffusion flames using flame stretch, $\chi_{s t}$, and flame temperature, $T_{s t}$, as coordinates. The solid black line corresponds to the extinction limit, $D a_{c}=1$. Reproduced from [8].

and/or thermal losses.

(2) non-premixed auto-ignition (an event controlled by both fuel-air mixing and chemistry); (3) edge flame propagation (a piloted ignition event controlled by 
partially-premixed flame propagation along the stoichiometric surface); and (4) flame-flame interactions (a piloted ignition event controlled by interactions of burning and extinguished flame elements normal to the stoichiometric surface).

Based on Hewson et al. [23], reignition mechanisms (1) and (2) (autoignition) are temperature controlled and require a temperature of approximately 1000 K. Reignition mechanisms (3) and (4) (piloted ignition) are not temperature controlled but may be less likely under the low-to-moderate turbulence conditions relevant to fire configurations.

In the absence of sufficient insight, in the present study, we choose to treat flame reignition via a critical gas temperature criterion,

$$
F I F=0.5+0.5 \tanh \left(\frac{\widetilde{T}-T_{i g n}}{100}\right)
$$

where FIF takes values between 0 and 1 and gives a measure of the local probability of flame reignition: $F I F=0$ if $\widetilde{T} \leqslant T_{i g n}$ (no reignition) and $F I F=$

${ }_{275} 1$ if $\widetilde{T} \geqslant T_{i g n}$ (reignition). In Eq. 11, $\widetilde{T}$ is the LES-filtered computational-cell temperature and $T_{i g n}$ its critical value for reignition. We use $T_{i g n}=1100 \mathrm{~K}$. It is worth emphasizing that the model in Eq. 11 is quite crude: it should be considered as a temporary placeholder that has merit because it acknowledges the need for an explicit treatment of reignition but also remains questionable because of limited physical insight.

\subsection{Numerical configuration}

The computational domain is presented in Fig. 3 and is 200-cm-wide in the cross-stream $x$-direction, 85 -cm-long in the spanwise $y$-direction and 200-cmhigh in the vertical $z$-direction. The grid is block-structured and features three levels of resolution (Fig. 3). The level-one refinement block contains the flame region and features a uniform grid with cubic cells; in the baseline case, the level-one grid cell size is $\Delta x_{1}=4.167 \mathrm{~mm}$ (i.e., 12 grid cells across the burner width). The level-one refinement block is 40-cm-wide, 60-cm-long and 60-cmhigh. The level-two refinement block features a uniform grid with cubic cells of size equal to $\left(2 \times \Delta x_{1}\right)=8.333 \mathrm{~mm}$; this block is 60 -cm-wide, 80 -cm-long and 


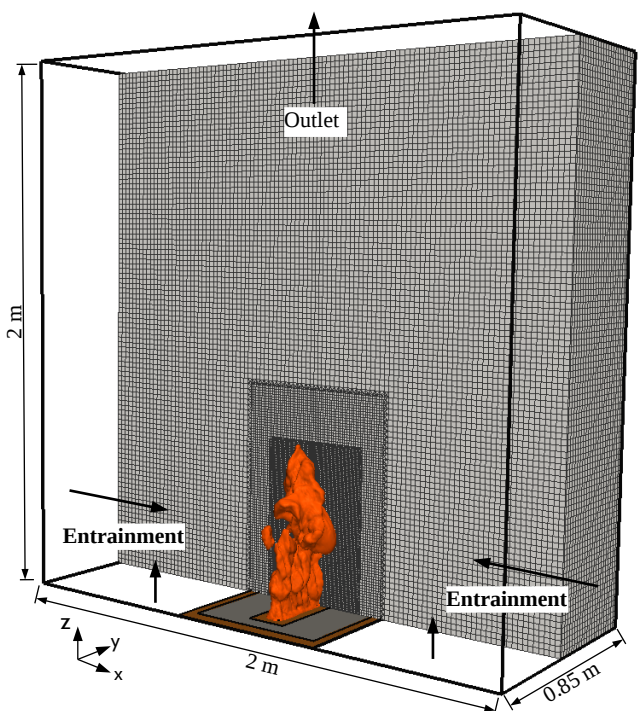

Figure 3: Computational domain and multi-level mesh refinement. The burner is $5 \mathrm{~cm}$ wide in the $x$-direction and $50 \mathrm{~cm}$ long in the $y$-direction; the flame height is approximately $50 \mathrm{~cm}$.

80-cm-high. The level-three refinement block covers the far-field air entrainment and plume regions and features a uniform grid with cubic cells of size equal to $\left(4 \times \Delta x_{1}\right)=16.67 \mathrm{~mm}$. The total number of cells is 1.76 million.

The inlet surfaces for fuel and oxidizer (at $z=0$ ) are treated as boundaries with a prescribed mass flow rate (including both convective and diffusive fluxes, see [13] for details). The inflow is assumed laminar. The ceramic fiberboard plate (at $z=0$ ) is treated as a no-slip adiabatic solid wall. The entrainment boundaries (at $x=-1$ and $1 \mathrm{~m}$ and $y=0$ and $0.85 \mathrm{~m}$ ) and the outlet boundary (at $z=2 \mathrm{~m}$ ) are treated as boundaries with open flow conditions.

300

All FireFOAM simulations in the present study are performed for a duration of $20 \mathrm{~s}$. Turbulent statistics are collected for the final $12 \mathrm{~s}$ of each simulation, after the flow and flame become statistically stationary and long enough for the first and second order turbulent statistics to be converged. The time step is 


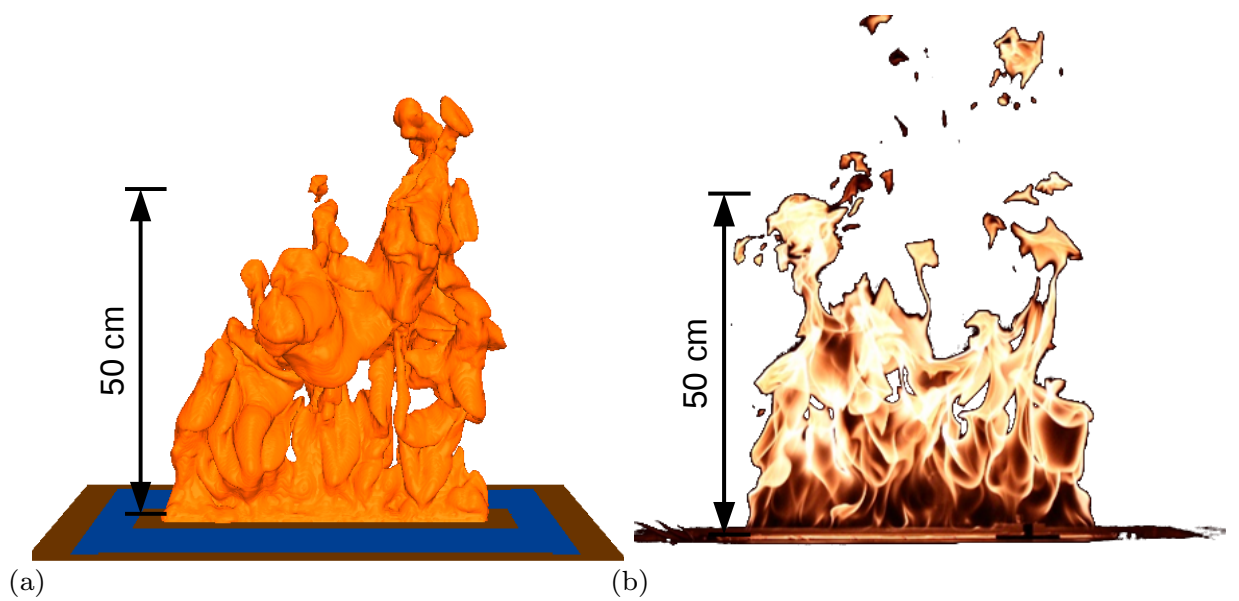

Figure 4: Instantaneous view of the flame surface; case with normal air co-flow, $X_{\mathrm{O}_{2}}=21 \%$. Comparison between: (a) the simulated flame surface defined as an iso-contour of the volumetric heat release rate; (b) the experimental flame surface obtained from a photographic view of the visible light emission from the flame.

controlled by a classical Courant-Friedrichs-Lewy (CFL) condition and in the baseline case, is equal to $0.5 \mathrm{~ms}$. Each simulation is run using 40 processors on a large-scale Linux cluster with typical runs requiring 2700 hours of CPU time. Note that the parallel performance of a solver is often measured by a characteristic time corresponding to the time consumed by a given simulation divided by the product of the total number of time steps times the total number of grid cells; in the present study, this characteristic time is approximately 140 us. 


\section{Results}

\subsection{Flame structure}

Using the baseline grid presented in the previous section $\left(\Delta x_{1}=4.167 \mathrm{~mm}\right)$, the simulations are found to provide a well-resolved description of the flame dynamics. Figure 4 presents a comparison between instantaneous views of the simulated and experimental flame surface in the case where the co-flow supplies normal air $\left(X_{\mathrm{O}_{2}}=21 \%, \chi_{r}=0.23, T_{s t}^{a d}=2240 \mathrm{~K}\right)$. The simulated flame surface is defined as an iso-contour of the volumetric heat release rate $\left(200 \mathrm{~kW} / \mathrm{m}^{3}\right)$. The experimental flame surface is obtained from a photographic view of the visible light emission from the flame region, thereby assuming that soot luminosity provides a suitable tracer of the flame contour.

The simulated and experimental flames feature a small laminar region at low elevations $(z<5 \mathrm{~cm})$ followed by a rapid transition to turbulent-like motions at higher elevations $(z>5 \mathrm{~cm})$. The flame height is approximately $50 \mathrm{~cm}$. Both the simulated and experimental flames show similar buoyancy-driven motions and in particular thin finger-like structures near the flame base that evolve into large intermittent structures near the flame tip. The spacing of the flamebase fingers is approximately 3 or $4 \mathrm{~cm}$ in the simulations and appears slightly smaller (between 1 and $2 \mathrm{~cm}$ ) in the experiment. This discrepancy may be due to insufficient grid resolution in the laminar-to-turbulent flow transition region (at $z<5 \mathrm{~cm}$ ). The spacing of the flame-tip peak and trough structures is approximately $10 \mathrm{~cm}$ in the simulations and is similar to that observed in the experiments.

35 Measured and simulated flame heights, noted $L_{f}$, are plotted as a function of $X_{\mathrm{O}_{2}}$ in Fig. 5. Starting from a flame with normal air co-flow, $X_{\mathrm{O}_{2}}=21 \%$, it is seen that nitrogen dilution (i.e., reduced $X_{\mathrm{O}_{2}}$ ) results in taller flames, both in the simulations and in the experiments. This tendency may be explained by the fact that as $X_{\mathrm{O}_{2}}$ decreases, a greater volume of oxidizer must be entrained by 340 the flame to support complete combustion: the flame must therefore lengthen to entrain the additional required oxidizer. As shown in Fig. 5, relatively good 


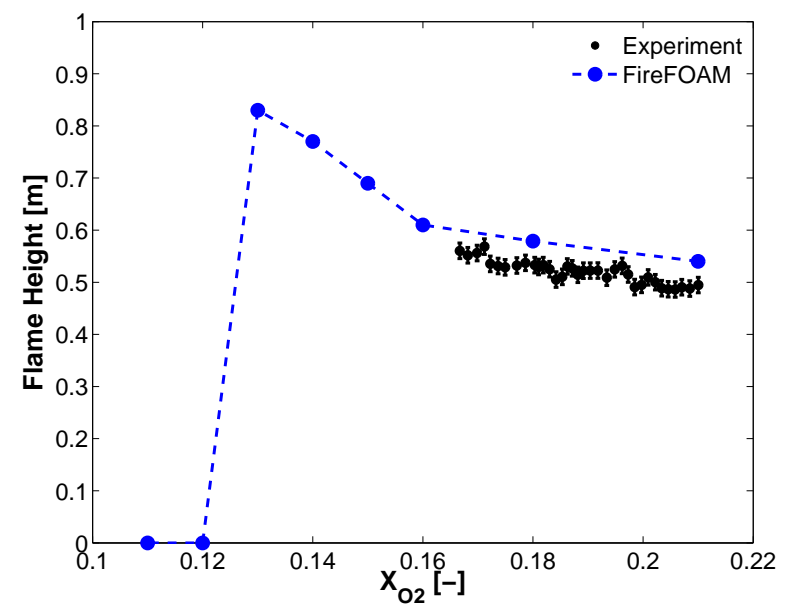

Figure 5: Measured (small dots) and simulated (large dots and dashed line) mean flame height as a function of $X_{O_{2}}$. Flames with normal air co-flow are on the right of the plot $\left(X_{\mathrm{O}_{2}}=21 \%\right)$; flames with strongly diluted nitrogen-air coflow are on the left. Simulated flames with $X_{\mathrm{O}_{2}}<X_{\mathrm{O}_{2} \text {, ext }}$ are fully suppressed (in the experiments, $X_{\mathrm{O}_{2}, e x t}=12.2 \%$ ).

agreement is obtained between simulated and measured values of $L_{f}$. It is worth noting, however, that the comparison in Fig. 5 remains somewhat qualitative due to differences in the definitions of the simulated and experimental flame heights. In the experiments, flame height is measured based on a $50 \%$ intermittency criterion for visible flame emission [9]. In the simulations, flame height is calculated based on averaging the maximum elevation of the instantaneous volumetric heat release rate distribution. Also, the experimental measurements of flame height are limited to the sooting regime of the flame $\left(X_{O_{2}}>16 \%\right)$ : for co-flows with low oxygen strength $\left(12.2 \% \leq X_{O_{2}} \leq 16 \%\right)$, the flame turns from yellow to blue and there is no longer sufficient contrast between the flame and the background to allow for an accurate visualization and flame height measurement; and, as previously mentioned (section 2.1), for co-flows with very low oxygen strength $\left(X_{\mathrm{O}_{2}}<12.2 \%\right)$, global flame extinction is observed. 

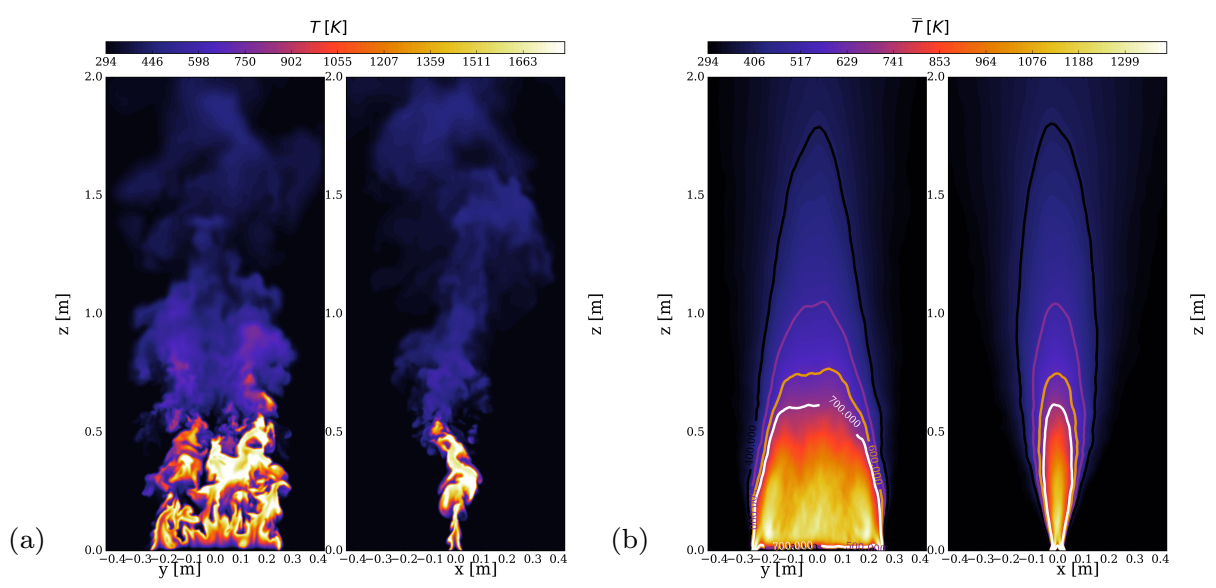

Figure 6: Front-view (left) and side-view (right) of the simulated temperature field in the central $(x-z)$ plane; case with normal air co-flow, $X_{O_{2}}=21 \%$. (a) Instantaneous distribution. (b) Time-averaged distribution. The colored solid lines correspond to different temperature iso-contours: $400 \mathrm{~K}$ (black), $500 \mathrm{~K}$ (red or grey), $600 \mathrm{~K}$ (orange or bright grey), and $700 \mathrm{~K}$ (white).

Figure 6 presents front- and side-views of the simulated instantaneous and time-averaged spatial distributions of temperature in the flame and plume regions. The peak values of the instantaneous temperature in the flame region are close to $1800 \mathrm{~K}$, which are reasonable values considering that the adiabatic flame temperature is $2240 \mathrm{~K}$ and that there is a $23 \%$ radiative heat loss. The peak value of the time-averaged temperature is close to $1300 \mathrm{~K}$. As shown in Fig. 6-(b), the mean flame width increases with elevation; at mid-flame height, the mean flame width is approximately $20 \mathrm{~cm}$. The instantaneous temperature distributions in Fig. 6-(a) feature the same kind of pattern already observed in Fig. 4: thin finger-like structures at the base of the flame evolving into larger peak and trough structures at the tip.

Figure 7 presents similar views of the simulated instantaneous and timeaveraged spatial distributions of vertical flow velocity. The peak values of the 

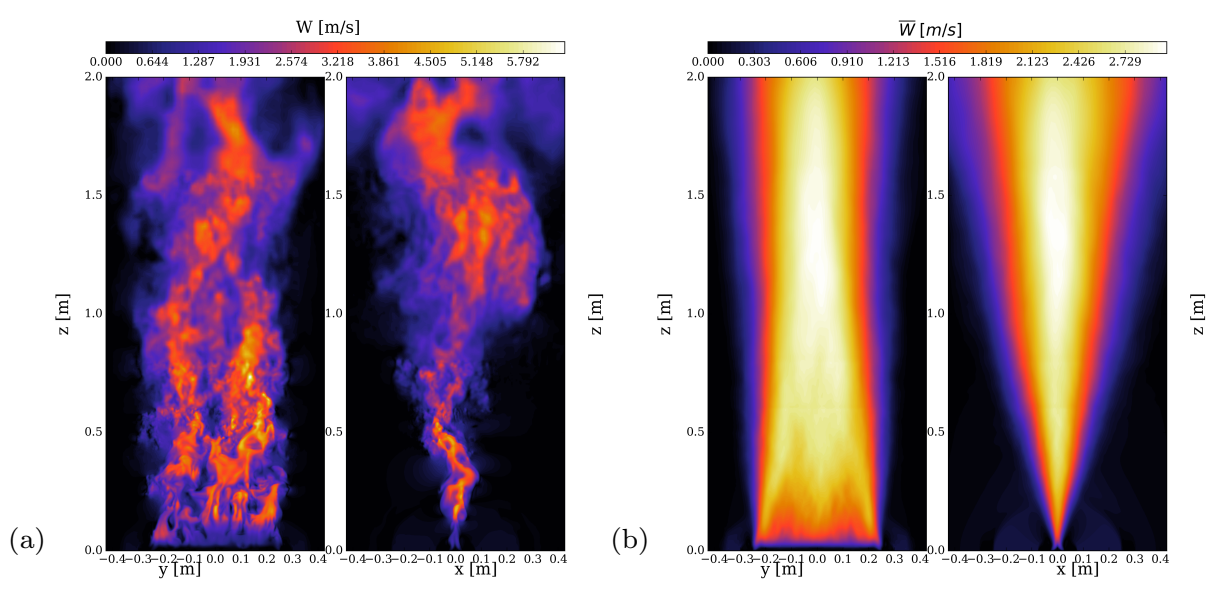

Figure 7: Front-view (left) and side-view (right) of the simulated vertical flow velocity field in the central $(x-z)$ plane; case with normal air co-flow, $X_{O_{2}}=$ $21 \%$. (a) Instantaneous distribution. (b) Time-averaged distribution.

instantaneous $z$-velocity are close to $6 \mathrm{~m} / \mathrm{s}$. The peak value of the time-averaged $z$-velocity is close to $3 \mathrm{~m} / \mathrm{s}$.

Figures 8,9 , and 10 adopt a different, more quantitative perspective and present the spatial variations of mean and root-mean-square $(r m s)$ quantities as a function of elevation $z$ along the flame centerline (figures (a)-(b)) and as a function of cross-stream distance $x$ at an elevation of $25 \mathrm{~cm}$ (corresponding to mid-flame height) (figures (c)-(d)). The mean quantities are obtained by direct time-averaging of the LES solution. The rms quantities are obtained by first calculating the fluctuation with respect to the local mean and then calculating the rms of the time variations of the fluctuation. Note that the $r m s$ quantities correspond to the grid-resolved amplitude of fluctuations, which may be different from the true amplitude; however, as will be seen in the next section, the present simulations are well-resolved and much of the fluctuation dynamics is captured by the simulations so that the reported rms quantities can be interpreted as estimates of the true values. 

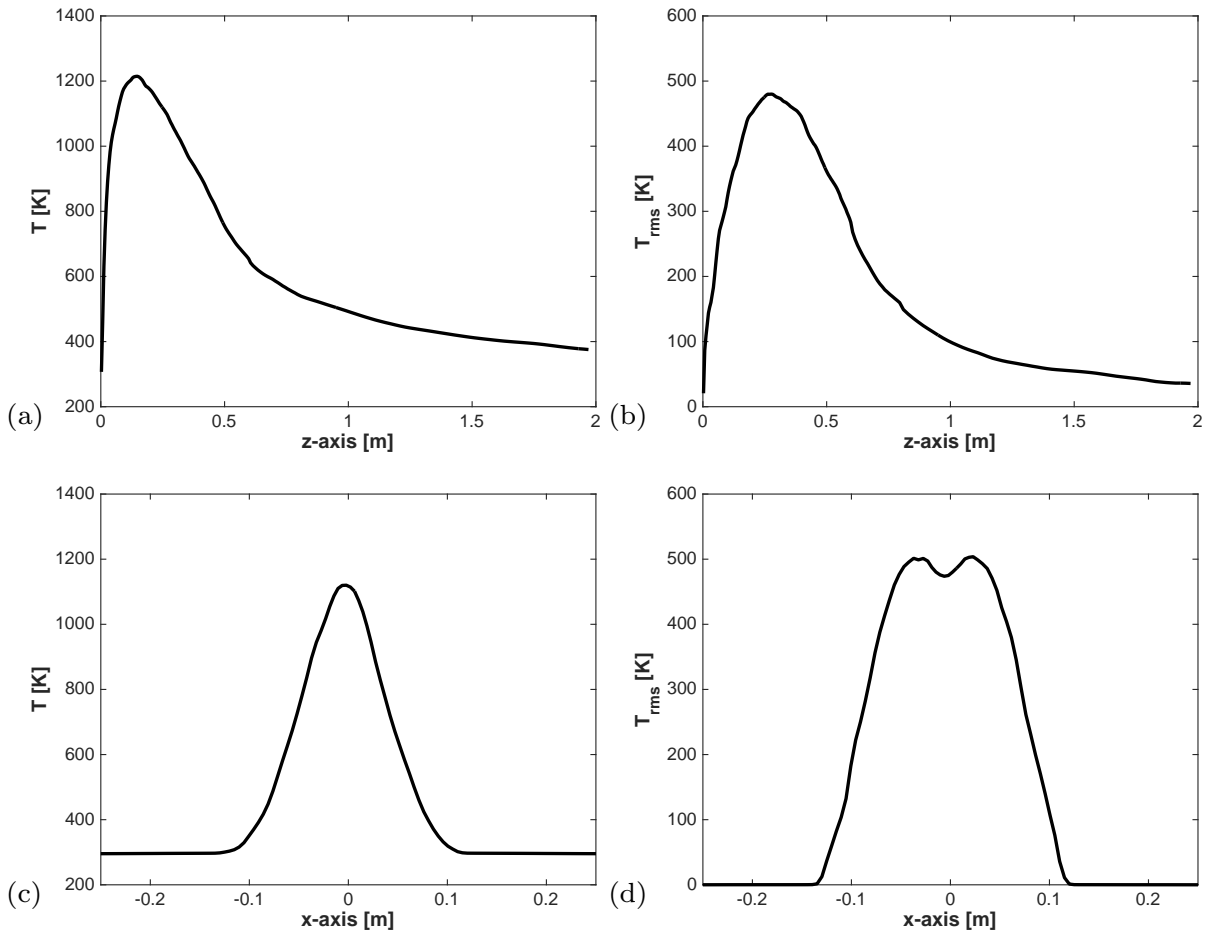

Figure 8: Spatial variations of simulated temperature; case with normal air co-flow, $X_{\mathrm{O}_{2}}=21 \%$. (a) Mean $z$-profile at $x=0$ (flame centerline). (b) rms $z$ profile at $x=0$. (c) Mean $x$-profile at $z=25 \mathrm{~cm}$. (d) rms $x$-profile at $z=25 \mathrm{~cm}$. Simulation with $\Delta x_{1}=4.167 \mathrm{~mm}$.

Figure 8 presents results for gas temperature. Figure 8-(a) shows that the mean temperature increases rapidly near the flame base to a peak value of $1200 \mathrm{~K}$ at an elevation of approximately $20 \mathrm{~cm}$ (close to mid-flame height) and then decays rapidly with an apparent change in the decay rate at an elevation of approximately $60 \mathrm{~cm}$ (close to flame height). Figure 8-(b) shows that the rms temperature follows similar trends and features a peak value of $480 \mathrm{~K}$ at an elevation of approximately $25 \mathrm{~cm}$. Thus, the peak value of the temperature fluctuation intensity is close to $40 \%$. Figure 8-(c)-(d) shows that the flame width 

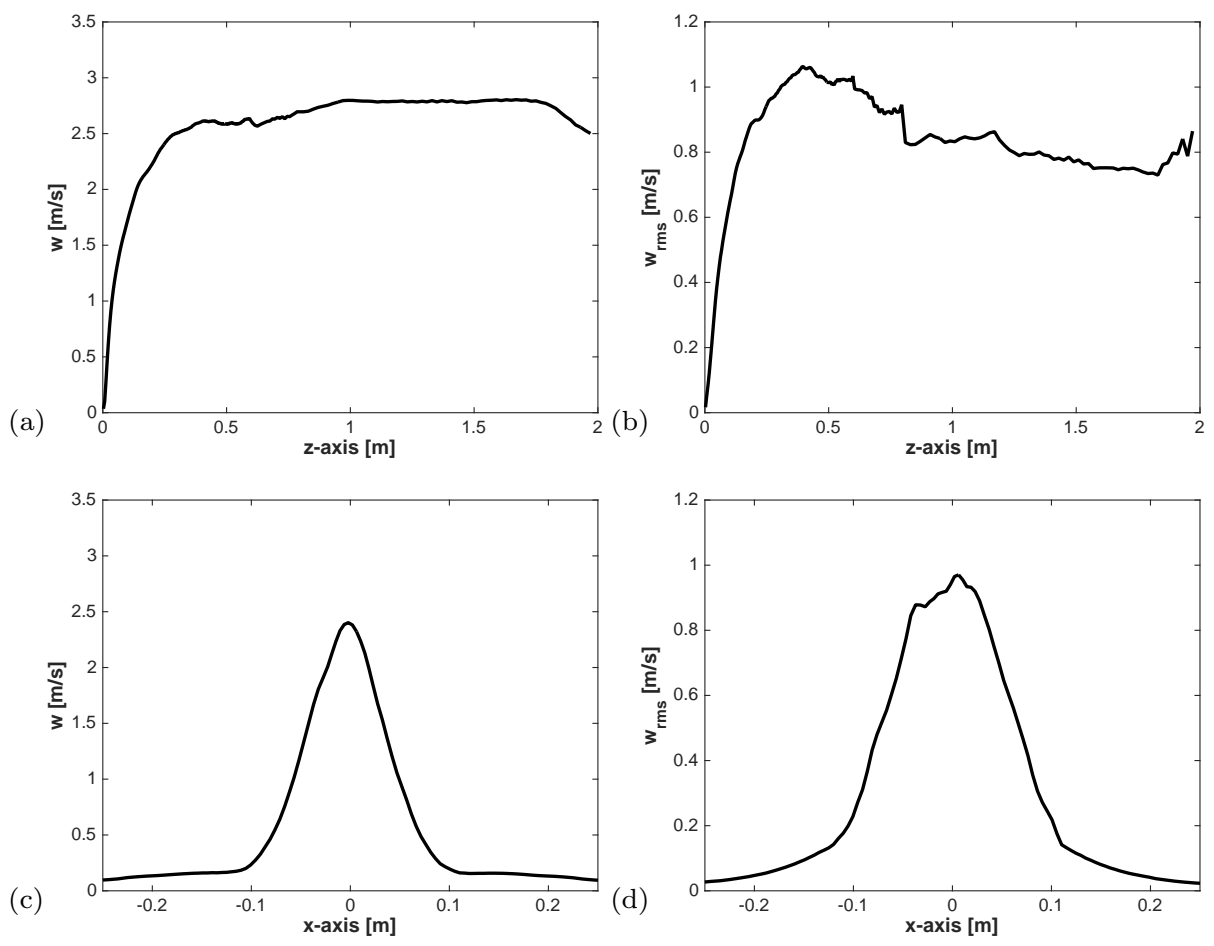

Figure 9: Spatial variations of simulated vertical flow velocity. See caption of Fig. 8 for details.

at mid-flame height is approximately $20 \mathrm{~cm}$. The slight double peak structure observed in Fig. 8-(d) is explained by the two flame sheets that develop at the lips of the burner and merge at the tip of the flame; the flame sheets are unsteady sources of heat and are responsible for strong fluctuations.

Figure 9 and 10 present similar results for the vertical and horizontal (crosstream) flow velocity, respectively. Figure 9-(a) shows that the mean vertical flow velocity increases rapidly near the flame base to a peak value of $2.6 \mathrm{~m} / \mathrm{s}$ at an elevation of approximately $35 \mathrm{~cm}$ (close to mid-flame height) and then stays approximately constant. Figure 9-(b) shows that the $r m s$ vertical flow velocity

follows similar trends and features a peak value of $1.1 \mathrm{~m} / \mathrm{s}$ at an elevation of ap- 

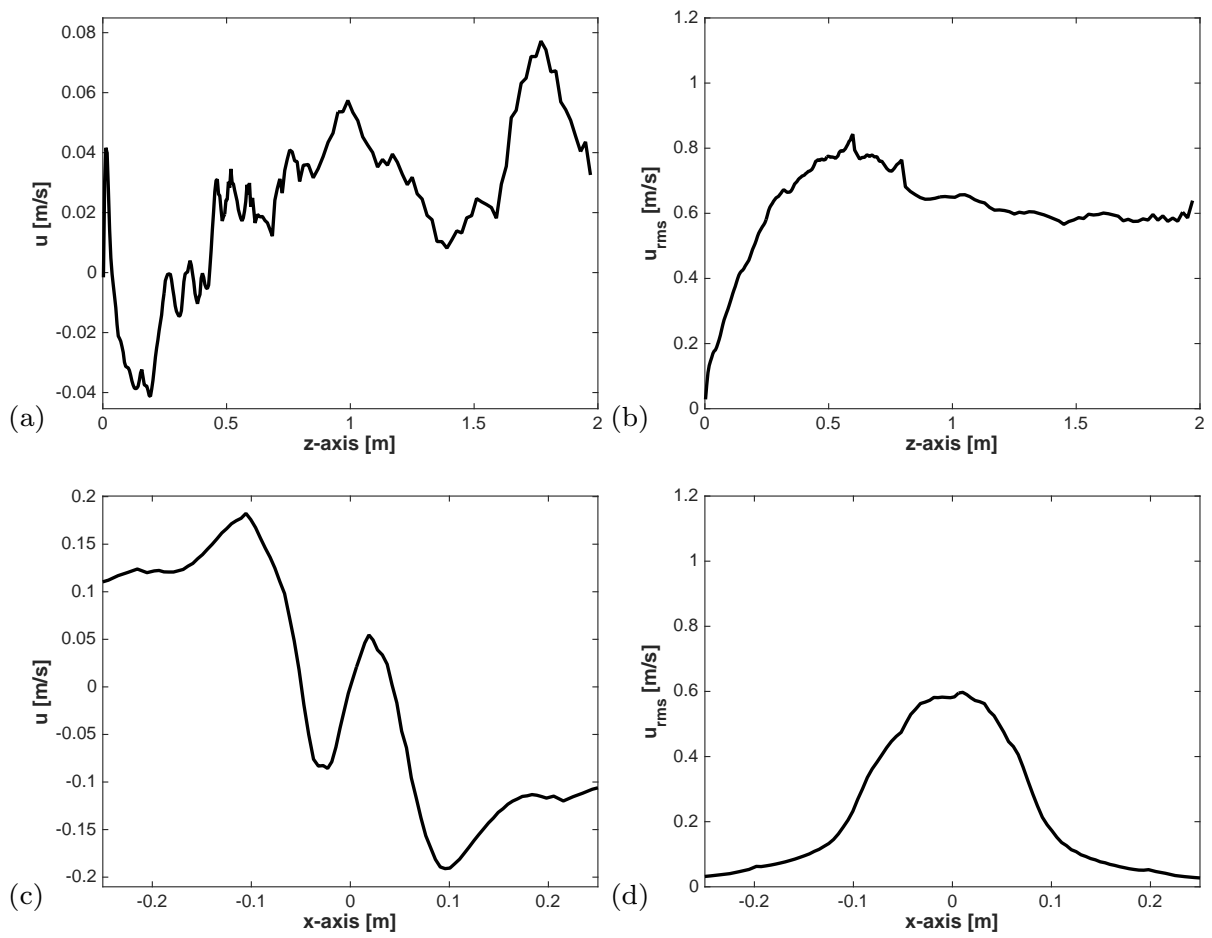

Figure 10: Spatial variations of simulated cross-stream flow velocity. See caption of Fig. 8 for details.

proximately $35 \mathrm{~cm}$. Thus, the peak value of the vertical flow velocity fluctuation intensity is close to $40 \%$. Furthermore, focusing now on the horizontal motions, Figs. 10-(c)-(d) show that the entrainment velocities outside of the flame region are characterized by modest mean values (close to $10 \mathrm{~cm} / \mathrm{s}$ at $z=25 \mathrm{~cm}$ ) and large fluctuation intensities. Note that Fig. 10-(a) is simply a check that on the flame centerline, the mean cross-stream velocity takes values close to the exact value of 0 (due to symmetry): this plot provides a measure of the uncertainties associated with the post-processing of the LES data. This level of uncertainty is deemed acceptable (note that it could be reduced by performing longer simulations which would provide better statistics). 

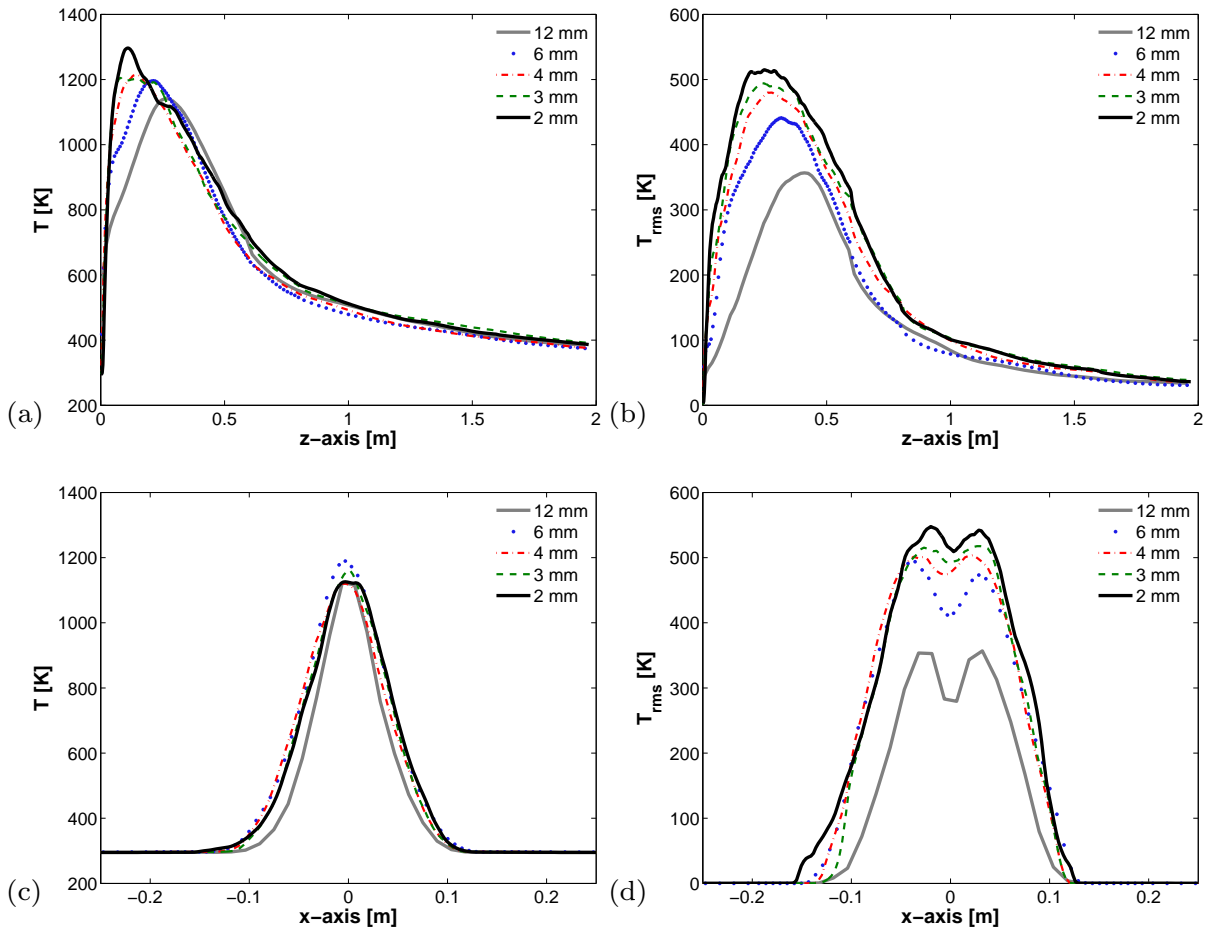

Figure 11: Spatial variations of simulated temperature. See caption of Fig. 8 for details. Grid convergence study: $\Delta x_{1}=2.083,3.125,4.167,6.25$, and $12.5 \mathrm{~mm}$.

It is also worth emphasizing that the simulated turbulent structure of the flame and plume regions presented in this section is in good agreement with classical results from the literature and in particular with previous experimental results on turbulent buoyant plumes in Ref. [28] and turbulent line burner flames in Ref. [29].

\subsection{Computational domain discretization (flow solver)}

The computational grid requirement in LES comes from the need to resolve the turbulent motions associated with energy-containing flow structures, also called the large eddies. The size of the large eddies is measured by the integral 

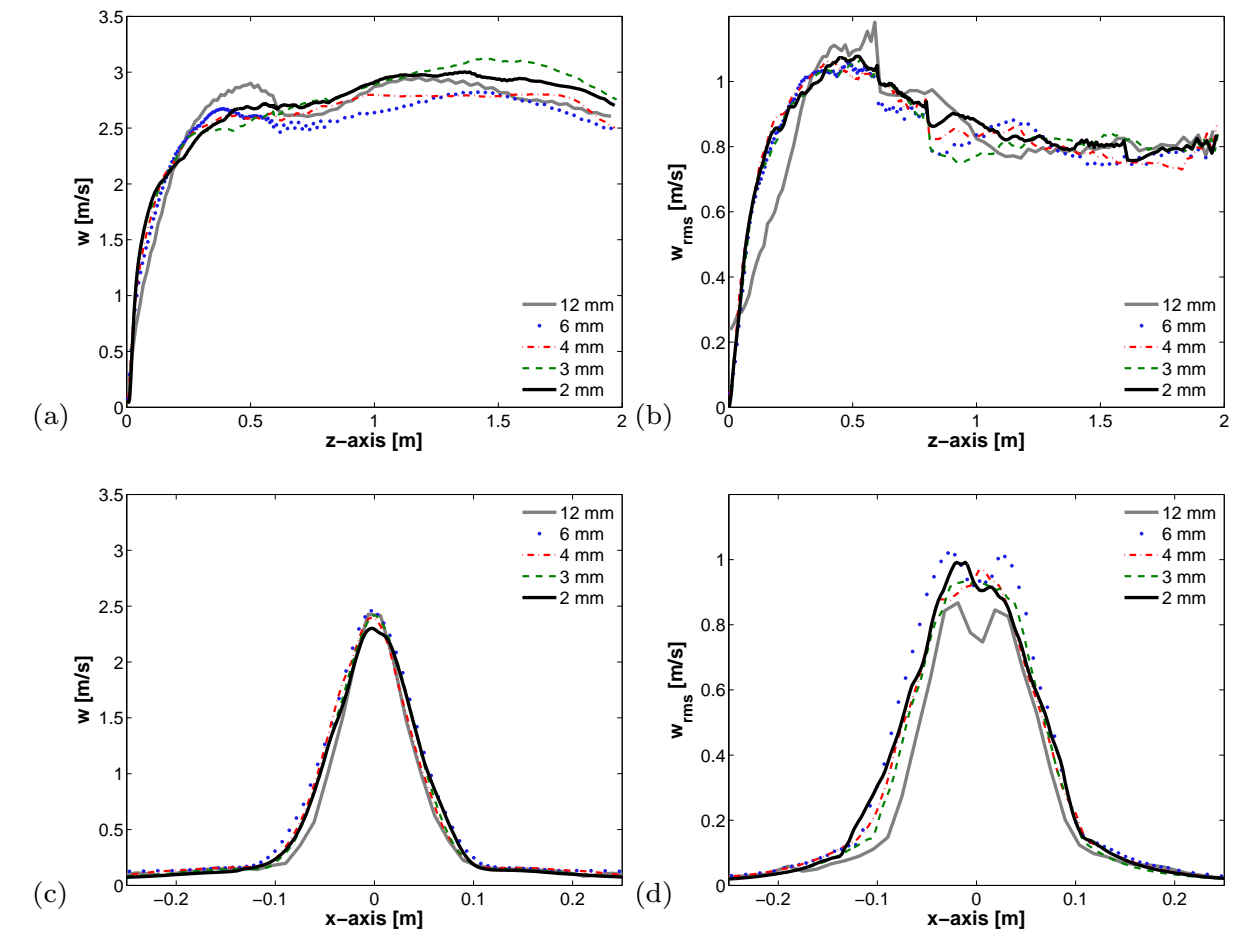

Figure 12: Spatial variations of simulated vertical flow velocity. See caption of Fig. 8 for details. Grid convergence study: $\Delta x_{1}=2.083,3.125,4.167,6.25$, and $12.5 \mathrm{~mm}$.

length scale of turbulence. Now, the integral length scale is not exactly known in the present configuration but is known to be related to the width of the flame or to that of the plume and an estimate is given by the width of the line burner, $W_{b}=5 \mathrm{~cm}$. In the baseline grid, the level-one grid resolution is $\Delta x_{1}=4.167 \mathrm{~mm}$ or $\left(W_{b} / \Delta x_{1}\right)=12$. In the following, we present a grid sensitivity analysis and consider additional grid resolutions corresponding to $\Delta x_{1}=2.083,3.125,6.25$, and $12.5 \mathrm{~mm}$, or $\left(W_{b} / \Delta x_{1}\right)=24,16,8$ and 4 . The total number of cells for the finest grid is 11.85 million.

Figures 11 and 12 consider the temperature and vertical flow velocity profiles 

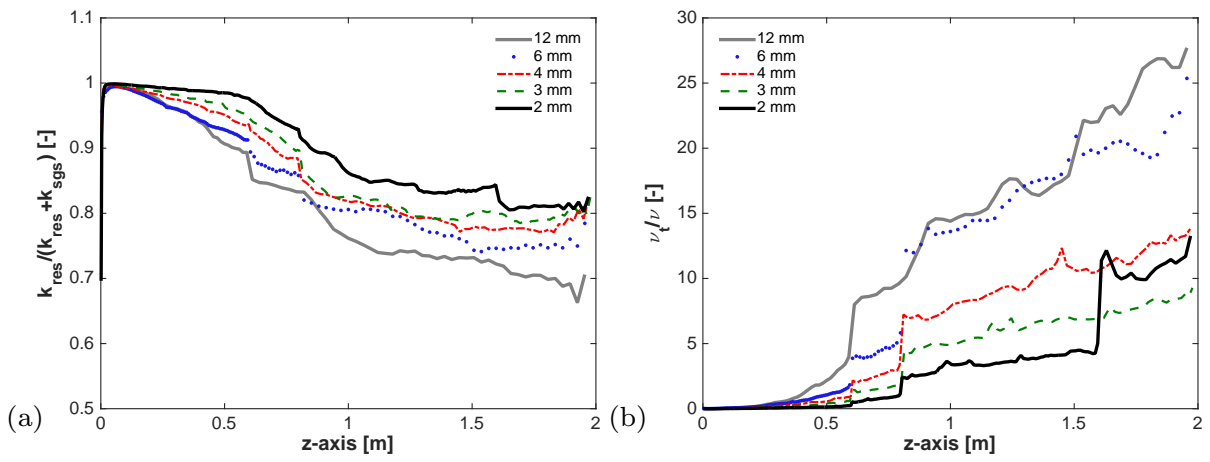

Figure 13: Spatial variations of LES resolution quality metrics along the flame centerline $(x=0)$; case with normal air co-flow, $X_{O_{2}}=21 \%$. (a) Fraction of resolved TKE, $\left(k_{r e s} /\left(k_{r e s}+k_{S G S}\right)\right)$. (b) Mean SGS-to-molecular viscosity ratio, $\left(\overline{\nu_{t} / \nu}\right)$. Grid convergence study: $\Delta x_{1}=2.083,3.125,4.167,6.25$, and $12.5 \mathrm{~mm}$.

already presented in Figs. 8 and 9 and compare the same profiles extracted from simulations performed at different grid resolutions. These plots show good convergence properties and suggest that the LES solutions become approximately grid-insensitive for $\Delta x_{1} \leq 4.167 \mathrm{~mm}$, although some slight variations in peak values persist (within 10\%) even at the highest levels of resolution.

Results in Figs. 11 and 12 suggest that the present LES simulations are gridconverged. A different perspective is adopted in Fig. 13. Following Pope [30], we define a well-resolved LES simulation as a simulation in which anywhere in the flow, the local ratio of the mean (i.e., time-averaged) grid-resolved turbulent kinetic energy $k_{r e s}$ divided by the mean total turbulent kinetic energy (equal to the sum of $k_{r e s}$ plus the mean subgrid-scale turbulent kinetic energy $k_{S G S}$ ) 440 is above $80 \%$. We also require that anywhere in the flow, the mean ratio of subgrid-scale eddy viscosity $\nu_{t}$ divided by molecular viscosity $\nu$ remains less than 1 [31]. If we first limit the discussion to the flow field, the subgrid-scale-eddyto-molecular viscosity ratio gives a convenient measure of the weight of subgrid- 
scale models in the LES flow solution and is an indicator of the distance between the grid resolution used in LES and the resolution that would be required in a DNS treatment. Now extending the discussion to include both the flow and scalar fields, small values of the viscosity ratio are required here to help provide approximately grid-independent solutions.

Figure 13-(a) shows that in all cases, more than $70 \%$ of the turbulent kinetic energy is grid-resolved in the plume region $(50 \mathrm{~cm}<z)$ and that this fraction increases to more than $90 \%$ inside the flame region $(z \leq 50 \mathrm{~cm})$. This result may be explained as follows: the turbulent structures observed in Figure 4 have a size of several centimeters and are therefore properly captured on a computational grid of size smaller than $10 \mathrm{~mm}$. From that perspective, the resolution quality 455 provided by the coarsest grid, $\Delta x_{1}=12.5 \mathrm{~mm}$, is expected to be only marginal. This point becomes more apparent when considering the mean subgrid-scaleeddy-to-molecular viscosity ratio. Figure 13 -(b) shows that in all cases, $\left(\overline{\nu_{t} / \nu}\right)$ takes values above 1 in the plume region and takes values below 1 in the flame region except for the two coarsest grids, $\Delta x_{1}=6.25 \mathrm{~mm}$ and $12.5 \mathrm{~mm}$. Thus, if one focuses on the flame region $(z \leq 50 \mathrm{~cm})$, the LES solutions are well-resolved and approximately grid-independent (in the sense defined above) provided that $\Delta x_{1} \leq 4.167 \mathrm{~mm}$.

Furthermore, note that some discontinuities can be seen in the profiles plotted in Fig. 13 at $z=60 \mathrm{~cm}$ and $z=80 \mathrm{~cm}$ : these discontinuities are the result of the variations in grid spacing at the interfaces between level-one and level-two refinement blocks, and level-two and level-three refinement blocks, respectively. In addition, the finest grid, $\Delta x_{1}=2.083 \mathrm{~mm}$, also features a level-four refinement block at high elevations, at $z>160 \mathrm{~cm}$, with cubic cells of size equal to $\left(8 \times \Delta x_{1}\right)=16.67 \mathrm{~mm}$, which explains the additional discontinuity seen for this case at $z=160 \mathrm{~cm}$.

Additional grid convergence tests (not shown here) were performed on resolution of the heat release rate variations, including near the flame extinction limit [22]. These tests were successful and confirmed that the baseline choice $\Delta x_{1}=4.167 \mathrm{~mm}$ allows for well-resolved simulations of the flame structure. All 
(a)

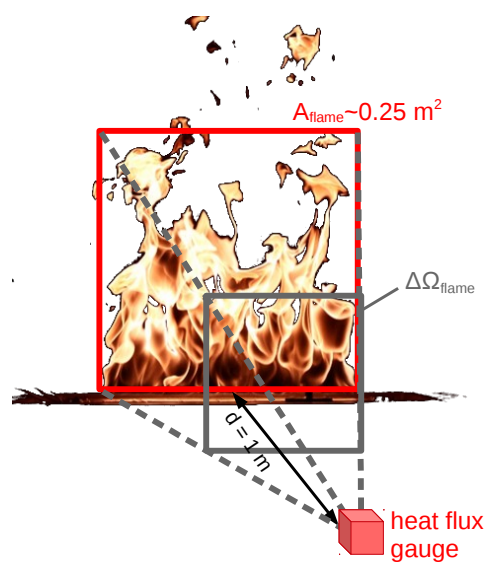

(b)

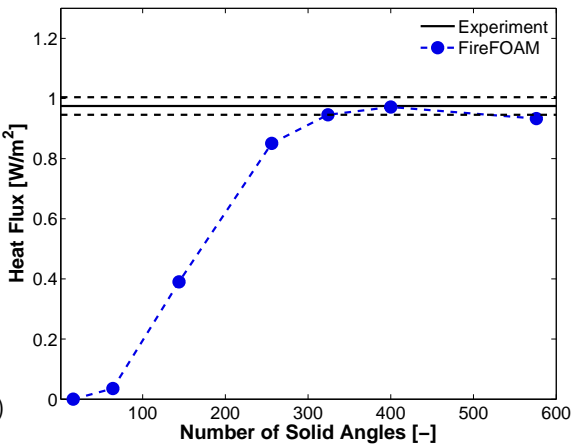

Figure 14: (a) Schematic of the experimental configuration for flame radiative emission measurements. (b) Simulated mean net heat flux at the remote gauge versus number of solid angles used in the RTE solver; case with normal air coflow, $X_{\mathrm{O}_{2}}=21 \%$. The horizontal solid line corresponds to the experimental measurement; the horizontal dashed lines mark the measurement uncertainty $( \pm 3 \%)$.

simulations in the following are performed with the baseline grid.

\subsection{Angular space discretization (radiation solver)}

In FireFOAM, the solver for the radiative transfer equation (RTE) is based on the discrete ordinate method and a uniform decomposition of angular space into $N_{\Omega}$ elementary solid angles, $\Delta \Omega=\left(4 \pi / N_{\Omega}\right)$. In the present simulations, the strongest requirement in the discretization of angular space comes from the need to resolve the radiation heat flux on the gauge used to measure the global radiative loss fraction [9]. This heat flux gauge is located at a distance of approximately $d=1 \mathrm{~m}$ from the flame in the cross-stream direction and is referred to in the following as the remote gauge. The surface area of the flame, as seen by the remote gauge, is approximately $A_{\text {flame }} \approx 0.25 \mathrm{~m}^{2}$, and the angular region occupied by the flame when viewed from the remote gauge may 
be estimated as $\Delta \Omega_{\text {flame }}=\left(A_{\text {flame }} / d^{2}\right)=0.25 \mathrm{sr}$ (see Fig. 14-(a)). It follows that an accurate representation of the radiative heat loading on the remote gauge requires an angular space discretization with $\Delta \Omega$ smaller than $\Delta \Omega_{\text {flame }}$ : ${ }_{490} \Delta \Omega=\left(\Delta \Omega_{\text {flame }} / N\right)$, where $N$ is an unknown number yet to be determined.

In this section, we present a sensitivity analysis and consider different levels of angular resolution corresponding to $\Delta \Omega=0.785,0.196,0.087,0.049,0.039$, 0.031 and $0.022 \mathrm{sr}\left(N_{\Omega}=16,64,144,256,324,400\right.$ and 576 solid angles $)$. Figure 14-(b) shows the variations of the simulated mean (time-averaged) value of the remote gauge heat flux for different levels of angular resolution. The quality of angular resolution is characterized by the number of solid angles $N_{\Omega}$. The data plotted in Fig. 14-(b) correspond to the case with normal air co-flow and also include the measured value of the heat flux. Figure 14-(b) shows that the simulated heat flux becomes approximately insensitive to $N_{\Omega}$, provided that $N_{\Omega} \geq 300$; the plot also shows that for $N_{\Omega} \geq 300$, the agreement between the simulated and measured heat flux is very good. These results suggest that a suitable description of the flame view angle is achieved for $\Delta \Omega \leq\left(\Delta \Omega_{\text {flame }} / 6\right)$. All simulations in the following are performed with $N_{\Omega}=324$.

\subsection{Flame radiative emissions}

As mentioned previously (section 2.1), the experimental database includes measurements of the global radiative loss fraction $\chi_{r}$ using a heat flux gauge (the remote gauge) combined with infrared camera imaging and a radiation source model [9]. It is found in Ref. [9] that the radiative loss fraction is not constant throughout the series of experiments and gradually decreases as the flame approaches its oxygen extinction limit. In the present study, the treatment of thermal radiation is simplified and directly uses the experimentally-determined values of $\chi_{r}$ as a function of the oxygen strength in the co-flow $X_{\mathrm{O}_{2}}$ (see Table $1)$.

We now perform a comparison between the simulated and measured mean values of the remote gauge heat flux as a function of $X_{\mathrm{O}_{2}}$ (Fig. 15). The results in Fig. 15 should not be interpreted as a validation test since the experimental 
Table 1: Prescribed values of the radiative fraction and adiabatic flame temperature for different dilution levels in the air-nitrogen co-flow $\left(X_{\mathrm{O}_{2}}\right)$.

\begin{tabular}{cll}
\hline$X_{\mathrm{O}_{2}}[\%]$ & $\chi_{r}[-]$ & $T_{s t}^{a d}[\mathrm{~K}]$ \\
\hline 21 & 0.23 & 2240 \\
18 & 0.18 & 1960 \\
16 & 0.145 & 1780 \\
15 & 0.12 & 1670 \\
14 & 0.12 & 1570 \\
13 & 0.12 & 1470 \\
12 & 0.12 & 1360 \\
11 & 0.12 & 1270 \\
\hline
\end{tabular}

data are used to prescribe flame radiative emissions in the numerical simulations but they may be interpreted as a consistency check. It is seen in Fig. 15 that the agreement between the simulated and measured heat flux is very good (less than $5 \%)$ for $X_{\mathrm{O}_{2}} \geq 16 \%$. For co-flows with low oxygen strength $\left(X_{\mathrm{O}_{2}}<14 \%\right)$, the flame weakens significantly and approaches full extinction: under those conditions, differences between the simulated and measured heat flux are due to differences between the simulated and measured extinction limits: $X_{O_{2}, e x t}=11 \%$ in the simulations and $X_{O_{2}, \text { ext }}=12.2 \%$ in the experiments (see the discussion below in section 3.6). Overall, Fig. 15 confirms that the baseline choice $N_{\Omega}=324$ allows for well-resolved simulations of the flame radiative emissions.

\subsection{Spatial variations of temperature and oxygen mole-fraction}

We now turn to a brief description of current results on spatial variations of temperature and oxygen mole-fraction statistics. The results currently available in the experimental database are preliminary, limited to mean (time-averaged) values, limited to cross-stream profiles at two elevations, and limited to the $X_{O_{2}}=18 \%$ condition. Future plans include an extension of the temperature measurements to mean and rms values using micro-thermocouples, to a more 


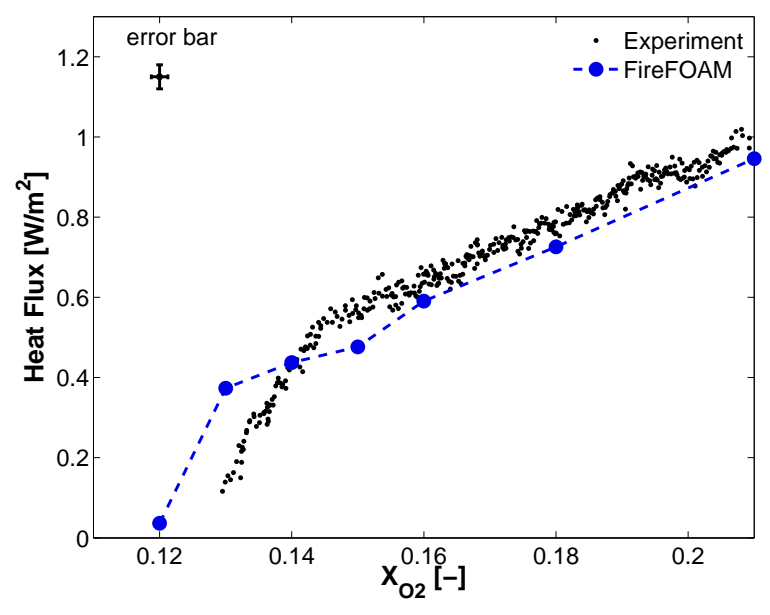

Figure 15: Comparison between simulated (large dots and dashed line) and measured (small dots) mean values of the net remote gauge heat flux for different levels of oxygen strength in the air-nitrogen co-flow.

complete map of the flame region, as well as an extension to cases with different nitrogen dilution conditions.

Figure 16 presents a comparison between the simulated and measured mean values of thermocouple temperature and oxygen mole-fraction as a function of cross-stream distance $x$ at an elevation of $12.5 \mathrm{~cm}$ (figures (a)-(b)) and an elevation of $25 \mathrm{~cm}$ (figures (c)-(d)). The simulated thermocouple temperatures are obtained using a thermocouple model in FireFOAM that accounts for bead size, bead thermal properties and convective and radiative heat exchanges with the surroundings [32]. The agreement between the simulated and measured data is good (less than 10\% ) although some limited discrepancies in peak value and width of the temperature profiles are observed in Figs. 16-(a) and 16-(c). This level of agreement suggests that both turbulent mixing and combustion heat release are simulated with good accuracy. 

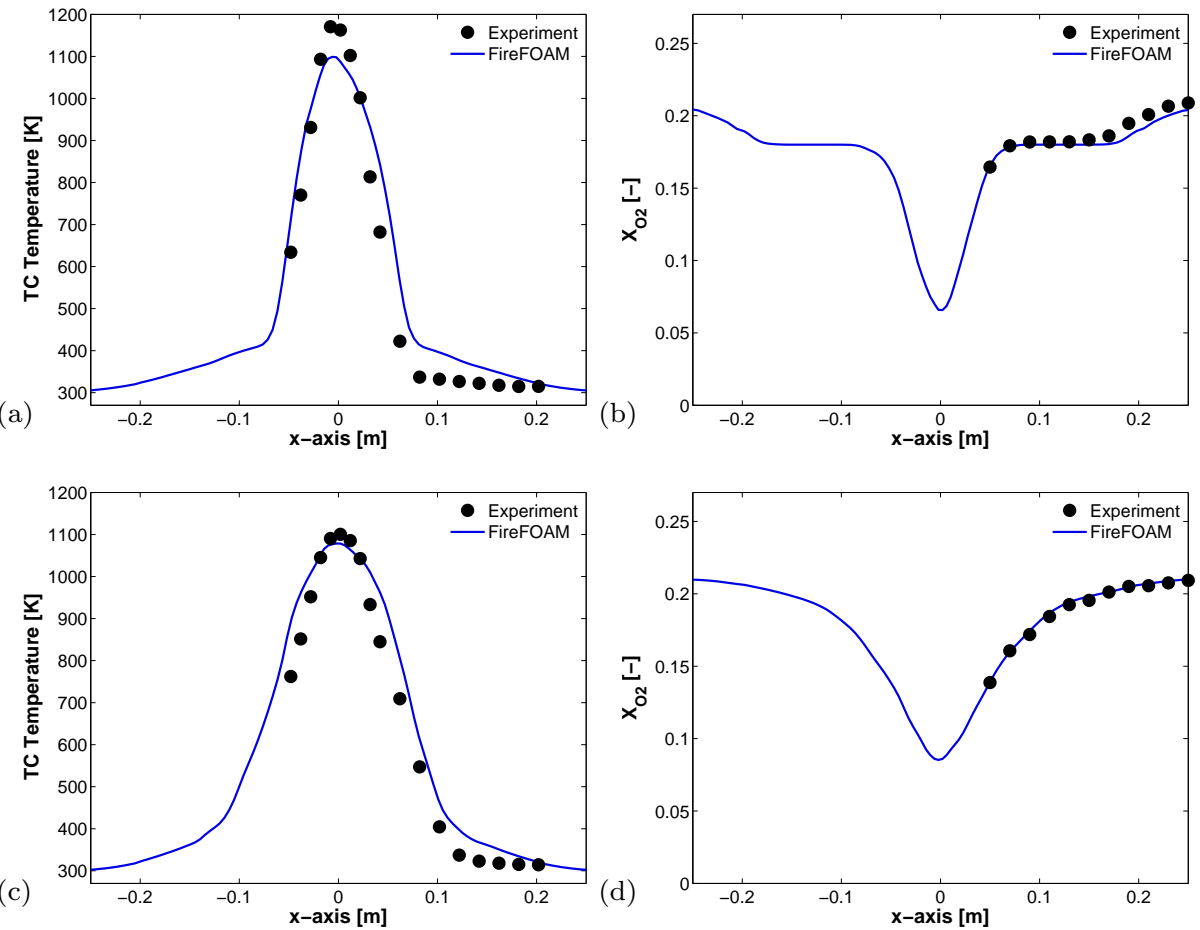

Figure 16: Spatial cross-stream variations of simulated (solid lines) and measured (dot symbols) mean values of temperature and oxygen mole-fraction; case with diluted air-nitrogen co-flow, $X_{O_{2}}=18 \%$. (a) Thermocouple temperature at $z=12.5 \mathrm{~cm}$. (b) Oxygen mole-fraction at $z=12.5 \mathrm{~cm}$. (c) Thermocouple temperature at $z=25 \mathrm{~cm}$. (d) Oxygen mole-fraction at $z=25 \mathrm{~cm}$

\subsection{Flame suppression}

This section examines the changes in combustion intensity and flame structure resulting from gradual reductions in the oxygen strength of the air-nitrogen co-flow. These experimentally observed changes allow an evaluation of the performance and accuracy of the flame extinction/reignition models presented in section 2.3 and correspond to the main focus of the present study. Flame weakening and transition to full extinction are characterized experimentally by mea- 

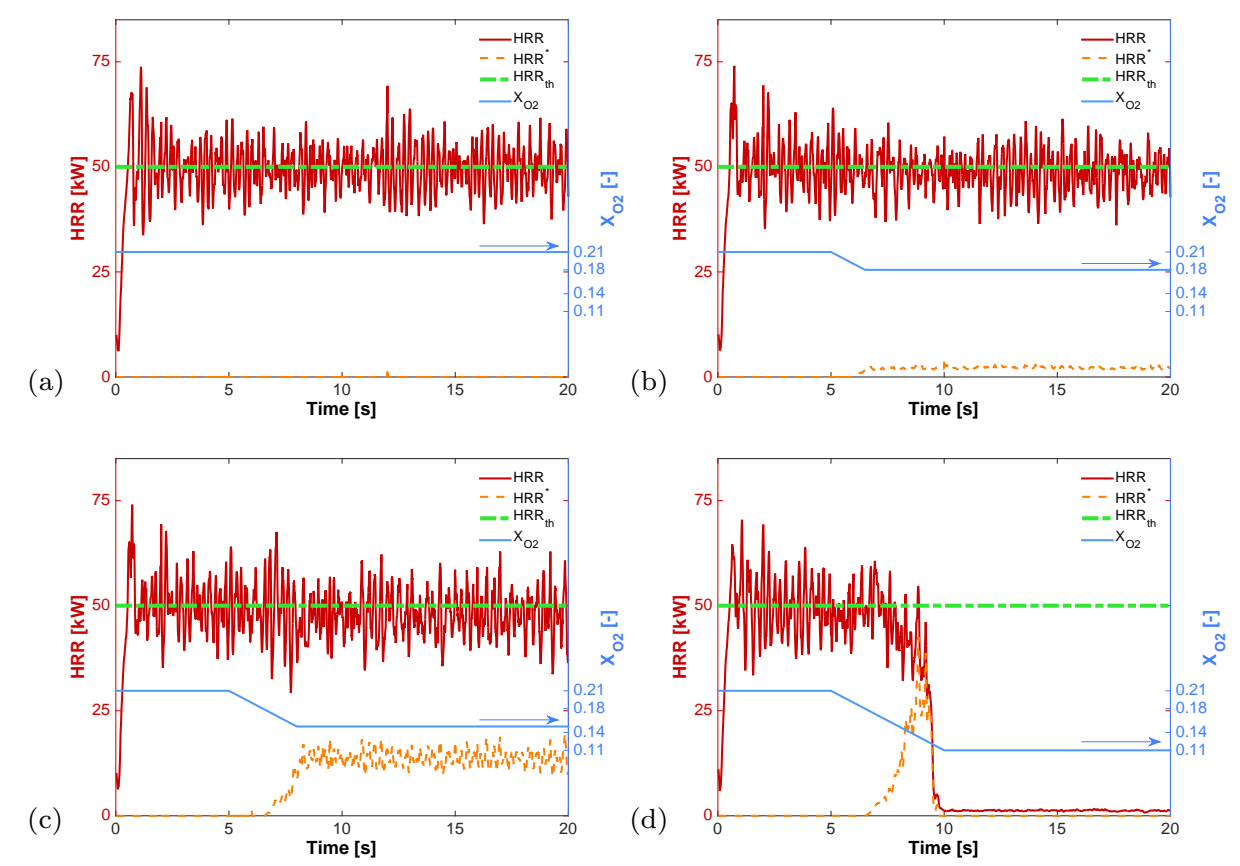

Figure 17: Simulated time variations of global $H R R$ (solid line) and $H R R^{*}$ (dashed line) under different co-flow conditions. (a) $X_{\mathrm{O}_{2}}=21 \%$. (b) $X_{\mathrm{O}_{2}}=$ $18 \%$. (c) $X_{\mathrm{O}_{2}}=15 \%$. (d) $X_{\mathrm{O}_{2}}=11 \%$. The upper horizontal dashed line corresponds to $H R R_{t h}$. The lower piecewise-linear solid line indicates the time variations of $X_{\mathrm{O}_{2}}$ (values to be read on the right vertical axis).

surements of the global combustion efficiency, noted $\eta_{\text {comb }}$ and defined as the ratio of total heat release rate divided by the product of the fuel mass flow rate $\dot{m}_{f}$ times the heat of combustion (per unit mass of fuel) $\Delta H_{f}$ :

$$
\eta_{c o m b}=\frac{\iiint_{V} \overline{\dot{\omega}_{h_{s}}^{\prime \prime \prime}} d V}{\left(\dot{m}_{f} \Delta H_{f}\right)}
$$

where the total heat release rate is expressed as the volume-integral of $\overline{\dot{\omega}_{h_{s}}^{\prime \prime \prime}}$ over a volume $V$ containing the flame region. Note that in the present study, the product $\left(\dot{m}_{f} \Delta H_{f}\right)$ is fixed and equal to $50 \mathrm{~kW}$. In the following, we write 
$H R R=\iiint_{V} \overline{\dot{\omega}_{h_{s}}^{\prime \prime \prime}} d V$ and $H R R_{t h}=\left(\dot{m}_{f} \Delta H_{f}\right)$

The measurements of $\eta_{c o m b}$ are essential to the discussion below and are performed in Ref. [10] using $\mathrm{CO}_{2}$ generation and $\mathrm{O}_{2}$ consumption calorimetry.

An alternative expression for the combustion efficiency is the ratio of the fuel mass reaction rate divided by the fuel mass flow rate $\dot{m}_{f}$ :

$$
\eta_{c o m b}=\frac{\iiint_{V} \overline{\dot{\omega}_{f}^{\prime \prime \prime}} d V}{\dot{m}_{f}}
$$
of local extinction and reignition phenomena: simulations predict $H R R^{*} \approx$ 
$3.5 \mathrm{~kW}$, which means that reaction (GR1) accounts for $93 \%$ of the heat release rate while reaction (GR3) accounts for $7 \%$.

Figure 17-(c) $\left(X_{\mathrm{O}_{2}}=15 \%\right)$ corresponds to a weakened flame. While the global combustion efficiency remains close to one, $\eta_{\text {comb }}=0.96$, the relative weight of local extinction and reignition phenomena is now much increased: simulations predict $H R R^{*} \approx 13.5 \mathrm{~kW}$, which means that reaction (GR1) accounts for $73 \%$ of the heat release rate while reaction (GR3) accounts for $27 \%$.

Finally, Figure 17-(d) $\left(X_{O_{2}}=11 \%\right)$ corresponds to a suppressed flame, $H R R \approx 0$ and $\eta_{c o m b} \approx 0$. Note that the values of $H R R$ and $\eta_{c o m b}$ are not exactly 0 at the end of the simulation: we find $H R R=1 \mathrm{~kW}$ and $\eta_{\text {comb }}=0.02$; these values correspond to a residual flame that is attached to, and maintained by the oxygen anchor. Note also that at the time of extinction, $t \approx 9 \mathrm{~s}$, the contribution of reaction (GR3) becomes dominant over that of reaction (GR1).

These results demonstrate that the flame extinction/reignition models implemented in FireFOAM are capable of describing (at least qualitatively) the transition from a strong flame with a global combustion efficiency close to one to a suppressed flame with a global combustion efficiency close to zero. We now turn to a more quantitative test and a comparison to experimental data on $\eta_{\text {comb }}$.

Figure 18 presents a comparison between simulated and measured values of the global combustion efficiency as a function of $X_{O_{2}}$. The experimental data show that the transition from a strong flame under normal air co-flow conditions to a suppressed flame under diluted air-nitrogen co-flow conditions is quite abrupt and occurs for $X_{\mathrm{O}_{2}}<14 \%$ [10]. The experimental extinction limit is $X_{O_{2}, e x t}=12.2 \%$. An important result is that the measured combustion efficiency remains close to one provided that $X_{\mathrm{O}_{2}} \geq 14 \%$. Figure 18-(a) shows that the numerical simulations correctly reproduce the main features of the response of the flame to nitrogen dilution and in particular the observed binary behavior of $\eta_{\text {comb }}: \eta_{c o m b} \approx 1$ for $X_{O_{2}} \geq 14 \%$ and $\eta_{\text {comb }} \approx 0$ for $X_{O_{2}}<12 \%$. The numerical extinction limit is $X_{O_{2}, e x t}=11 \%$, which means that the transition from a fully burning to a fully extinguished flame is slightly smoother in the 

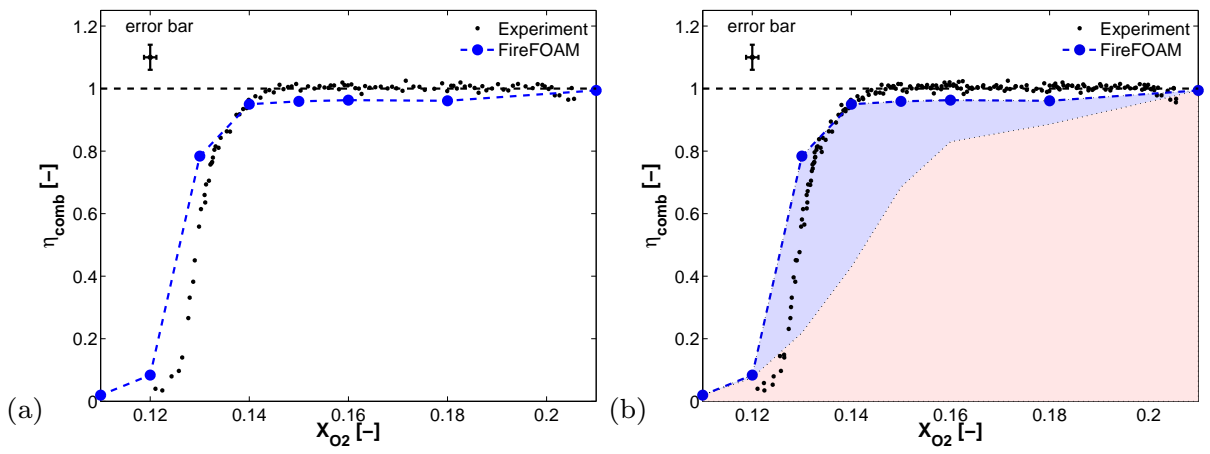

Figure 18: a) Comparison between simulated (large dots and dashed line) and measured (small dots) values of the global combustion efficiency, $\eta_{\text {comb }}$, for different levels of oxygen strength in the air-nitrogen co-flow. b) Relative contributions of reaction (GR1) (red color or light grey) and reaction (GR3) (blue color or dark grey) to $\eta_{c o m b}$. The horizontal dashed line corresponds to complete combustion, $\eta_{c o m b}=1.0$.

simulations than in the experiments.

Figure 18-(b) shows the relative contributions of reactions (GR1) and (GR3) to the heat release rate. This plot provides insight into the inner workings of the model but unfortunately cannot be validated by experimental data. The plot suggests that while the contribution of flame reignition is negligible at $X_{\mathrm{O}_{2}}=21 \%$, it becomes dominant for $X_{\mathrm{O}_{2}}<14 \%$, i.e. near the extinction limit. Thus it is found that the flame reignition model plays a central role in the simulated flame response and quenching behavior.

The results presented in Fig. 18 are quite encouraging. However, it is worth noting that the level of agreement between simulation results and experimental data depends on a number of factors including the values adopted for important model parameters. We now conclude this section by re-visiting some of the choices made in selecting values for key model parameters and focus in particular on the critical value of the flame Damköhler number at extinction, $D a_{c}=1$, and 

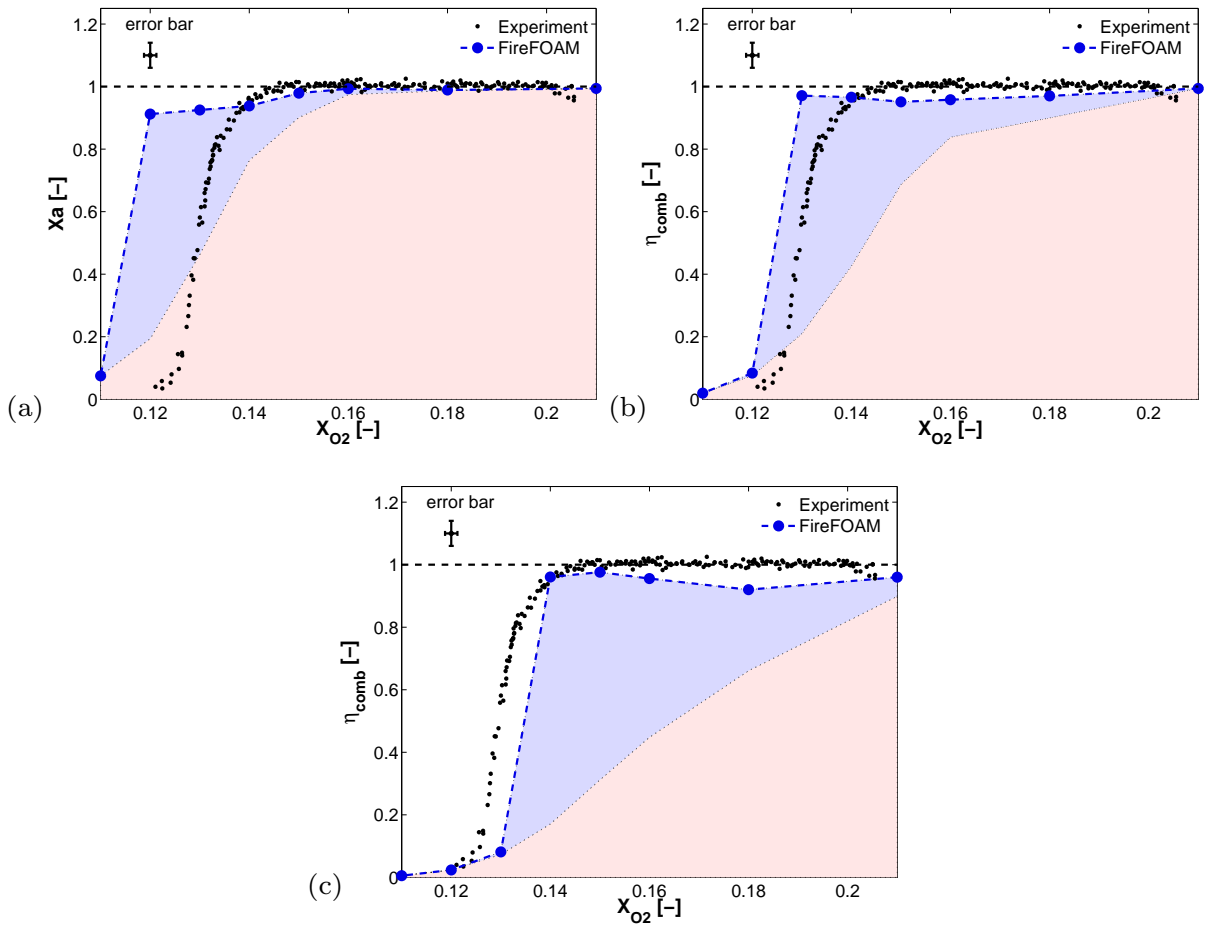

Figure 19: Comparison between simulated (large dots and dashed line) and measured (small dots) variations of $\eta_{c o m b}$ with $X_{O_{2}}$. (a) $D a_{c}=0.1$. (b) $D a_{c}=$ 1. (c) $D a_{c}=10$. The figures also show the relative contributions of reaction (GR1) (red color or light grey) and reaction (GR3) (blue color or dark grey) to $\eta_{c o m b}$. The horizontal dashed line corresponds to complete combustion, $\eta_{c o m b}=$ 1.0 .

the critical value of gas temperature at reignition, $T_{i g n}=1100 \mathrm{~K}$. Figures 19 and 20 examine the sensitivity of the simulated flame response to variations in the values of $D a_{c}$ and $T_{i g n}$, respectively (the model parameters $C$ and $T_{a}$ are kept unchanged in these tests).

Figure 19 presents the variations of $\eta_{c o m b}$ with $X_{O_{2}}$ for $D a_{c}=0.1,1$ and 10, and using $T_{i g n}=1000 \mathrm{~K}$. As seen in Fig. 19, the simulated extinction performance is strongly dependent on $D a_{c}$. For $D a_{c}=0.1$ (Fig. 19-(a)), the 

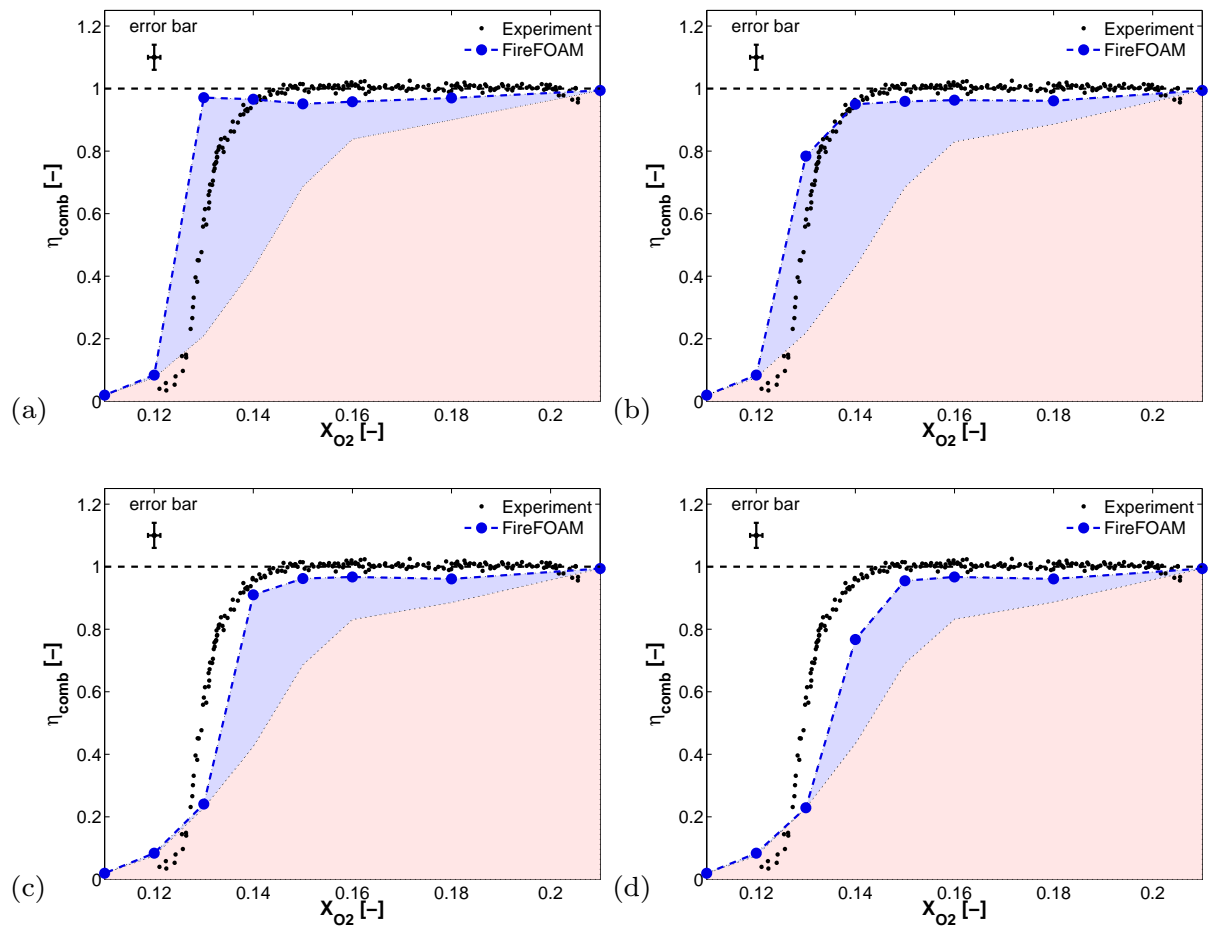

Figure 20: (a) $T_{i g n}=1000 \mathrm{~K}$. (b) $T_{i g n}=1100 \mathrm{~K}$. (c) $T_{i g n}=1200 \mathrm{~K}$. (d) $T_{i g n}=$ 1300 K. See caption of Fig. 19 for details.

640

flame is more resistant to extinction and $X_{O_{2}, e x t}<11 \%$. In contrast, for $D a_{c}=$ 10 (Fig. 19-(c)), the flame is less resistant to extinction and $X_{\mathrm{O}_{2}, \text { ext }} \approx 12 \%$. In this case, while the numerical extinction limit agrees with the experimental data $\left(X_{\mathrm{O}_{2}, \text { ext }}=12.2 \%\right)$, the model predicts partial extinction under normal air co-flow conditions, $\eta_{c o m b}<1.0$ and $H R R^{*}>0$ for $X_{O_{2}}=21 \%$, which does not seem correct. The best results are obtained for $D a_{c}=1$ (Fig. 19-(b)); the results are similar to those shown in Fig. 18 with the difference that in the present test, the reignition temperature has been changed from $1100 \mathrm{~K}$ to $1000 \mathrm{~K}$. This analysis suggests that the baseline choice $D a_{c}=1$ provides the best agreement with experimental data. 

and $1300 \mathrm{~K}$, and using $D a_{c}=1$. As seen in Fig. 20, the simulated extinction performance is only weakly dependent on $T_{i g n}$ for $X_{O_{2}} \geq 0.16$, probably due to the limited impact of flame reignition under those conditions. The simulated extinction performance is more sensitive to $T_{i g n}$ for $X_{\mathrm{O}_{2}}<0.16$. For ${ }_{655} T_{i g n}=1000 \mathrm{~K}$ (Fig. 20-(a)), the flame is less resistant to reignition, the relative contribution of reaction (GR3) is increased, and the transition from a fully burning to a fully extinguished flame is more abrupt. In contrast, for $T_{i g n}=1300 \mathrm{~K}$ (Fig. 20-(d)), the flame is more resistant to reignition and the transition from a fully burning to a fully extinguished flame is smoother. The best results are obtained for $T_{i g n}=1100 \mathrm{~K}$ (Fig. 20-(b)) or $T_{i g n}=1200 \mathrm{~K}$ (Fig. 20-(c)). This analysis suggests that the baseline choice $T_{i g n}=1100 \mathrm{~K}$ is adequate.

\section{Conclusion}

This paper presents a numerical study of a buoyancy-driven, methane-fueled, turbulent diffusion flame exposed to an air-nitrogen co-flow with variable composition, including conditions for which the nitrogen displacement of oxygen leads to full flame extinction. Numerical results are compared to experimental data on global combustion efficiency and to preliminary data on local variations of temperature and oxygen mole-fraction. The comparison is aimed at evaluating new flame extinction/reignition models recently implemented in a LES-based fire simulation solver called FireFOAM. The models are based on the concepts of a critical flame Damköhler number for extinction and a critical gas temperature for reignition. It is found that the numerical simulations correctly reproduce the measured trend in global combustion efficiency and in particular the experimentally observed abrupt transition from a strong flame 675 $X_{O_{2}, e x t}=12.2 \%$; the numerical extinction limit is $X_{O_{2}, e x t}=11 \%$.

These results are obtained using well-resolved and approximately grid-independent 
LES simulations. The baseline choice for the computational grid provides 12 supercomputing resources (http://www.it.umd.edu/hpcc) made available in conducting the research reported in this paper. This work also used the Extreme Science and Engineering Discovery Environment (XSEDE), which is supported by a National Science Foundation grant (number TG-CTS140046). 
[1] Z. Hu, Y. Utiskul, J. G. Quintiere, A. Trouvé, Towards large eddy simulations of flame extinction and carbon monoxide emission in compartment fires, Proc. Combust. Inst. 31 (2007) 2537-2545.

[2] J. Vaari, J. Floyd, R. McDermott, CFD simulations on extinction of co-flow diffusion flames, Fire Safety Science, Proc. Tenth International Symposium, International Association for Fire Safety Science (2011) 781-793.

[3] J. C. Hewson, S. R. Tieszen, W. D. Sundberg, P. E. Desjardin, CFD modeling of fire suppression and its role in optimizing suppressant distribution, National Insitute of Standards and Technology, Special Publication (NIST SP)-984-4 (2003) .

[4] S. Vilfayeau, N. Ren, Y. Wang, A. Trouvé, Numerical simulation of under-ventilated liquid-fueled compartment fires with flame extinction and thermally-driven fuel evaporation, Proc. Combust. Inst. 35 (2015) 25632571 .

[5] A. Y. Snegirev, A. S. Tsoy, Treatment of local extinction in CFD fire modeling, Proc. Combust. Inst. 35 (2015) 2519-2526.

[6] A. Y. Snegirev, Perfectly stirred reactor model to evaluate extinction of diffusion flame, Combust. Flame 162 (2015) 3622-3631.

[7] V. Sankaran, H. Jiang, M. Colket, S. Zhang, M. Corn, Perfectly stirred reactor model to evaluate extinction of diffusion flame, Proc. 9th U.S. National Combustion Meeting, Cincinnati (2015) .

[8] V. Lecoustre, P. Narayanan, H. Baum, A. Trouvé, Local extinction of diffusion flames in fires, Fire Safety Science, Proceedings of the 11th International Symposium (2011) 583-595.

730

[9] J. P. White, E. D. Link, A. C. Trouvé, P. B. Sunderland, A. W. Marshall, J. A. Sheffel, M. L. Corn, M. B. Colket, M. Chaos, H.-Z. Yu, Radia- 
tive emissions measurements from a buoyant, turbulent line flame under oxidizer-dilution quenching conditions, Fire Safety J. 76 (2015) 74-84.

[10] J. P. White, E. D. Link, A. C. Trouvé, P. B. Sunderland, A. W. Marshall, A general calorimetry framework for measurement of combustion efficiency in a suppressed turbulent line fire, Fire Safety J. (2016), submitted for publication.

[11] FM Global, FireFOAM, available at: <https://github.com/ fireFoam-dev> (accessed: 11-30-2015).

[12] J. P. White, Measurement and simulation of suppression effects in a buoyant turbulent line fire, Ph.D. thesis, University of Maryland, College Park (2016).

[13] Y. Wang, P. Chatterjee, J. L. de Ris, Large eddy simulation of fire plumes, Proceedings of the Combustion Institute 33 (2) (2011) 2473-2480.

[14] Y. Wang, K. V. Meredith, X. Zhou, P. Chatterjee, Y. Xin, M. Chaos, N. Ren, S. B. Dorofeev, Numerical simulation of sprinkler suppression of rack storage fires, Fire Safety Science, Proc. Eleventh International Symposium, International Association for Fire Safety Science (2014) 1170-1183.

[15] OpenCFD Ltd., OpenFOAM, available at: <http://www.openfoam.com> (accessed: 11-30-2015).

[16] C. Fureby, G. Tabor, H. G. Weller, A. D. Gosman, A comparative study of subgrid scale models in homogeneous isotropic turbulence, Phys. Fluids 9 (1997) 1416-1429.

[17] B. Magnussen, B. Hjertager, On mathematical modeling of turbulent combustion with special emphasis on soot formation and combustion, Proc. Combust. Inst. 16 (1976) 719-729.

[18] J. Chai, P. Rath, Discretes-ordinates and finite-volume methods for radiation heat transfer, International Workshop on Discrete-Ordinates and Finite-Volume Methods for Radiation Heat Transfer (2006) . 
[19] J. P. White, S. Vilfayeau, A. W. Marshall, A. Trouvé, R. J. McDermott, Modeling flame extinction and reignition in large eddy simulations with fast chemistry, Fire Safety J. (2016) , submitted for publication.

[20] S. H. Chan, J. Q. Yin, B. J. Shi, Structure and extinction of methane-air flamelet with radiation and detailed chemical kinetic mechanism, Combust. Flame 112 (1998) 445-456.

[21] P. Narayanan, H. Baum, A. Trouvé, Effect of soot addition on extinction limits of luminous laminar counterflow diffusion flames, Proc. Combust. Inst. 33 (2) (2011) 2539-2546.

[22] S. Vilfayeau, Large eddy simulation of fire extinction phenomena, PhD

[23] J. C. Hewson, A. R. Kerstein, Local extinction and reignition in nonpremixed turbulent CO/H2/N2 jet flames, Combust. Sci. Technol. 174 (2002) 35-66.

[24] H. Pitsch, C. Cha, S. Fedotov, Flamelet modelling of non-premixed tur-

[26] R. Venugopal, J. Abraham, A 2-D DNS investigation of extinction and reignition dynamics in non-premixed flamevortex interactions, Combust. Flame 153 (3) (2008) $442-464$.

[27] D. O. Lignell, J. H. Chen, H. A. Schmutz, Effects of damköhler number on flame extinction and re-ignition in turbulent non-premixed flames using bulent combustion with local extinction and reignition, Combust. Theor. Model., 7 (2) (2003) 317-332.

[25] P. Sripakagorn, S. Mittarai, G. Kosaly, H. Pitsch, Extinction and reignition in a diffusion flame: a direct numerical simulation study, J. Fluid Mech. 518 (2004) 231-259.

DNS, Combust. Flame 158 (5) (2011) 949 - 963. 
[28] A. Shabbir, W. K. George, Experiments on a round turbulent buoyant plume, J. Fluid Mech. 275 (1994) 1-32.

[29] L.-M. Yuana, G. Cox, An experimental study of some line fires, Fire Safety J. 27 (2) (1996) 123-139.

[30] S. Pope, Turbulent Flows, Cambridge University Press, Cambridge, 2000.

[31] S. Kenjeres, K. Hanjalic, LES, T-Rans and hybrid simulations of thermal convection at high $R a$ numbers, International Journal of Heat and Fluid Flow 27 (2006) 800-810.

[32] N. Ren, Y. Wang, S. Vilfayeau, A. Trouvé, Large eddy simulation of turbulent vertical wall fires supplied with gaseous fuel through a porous burner, Combust. Flame (2015), accepted. 